\title{
Particle-in-cell experiments examine electron diffusion by whistler-mode waves: 1. Benchmarking with a cold plasma
}

Article

Accepted Version

Allanson, O., Watt, C., Ratcliffe, H., Meredith, N., Allison, H., Bentley, S., Bloch, T. and Glauert, S. (2019) Particle-in-cell experiments examine electron diffusion by whistler-mode waves: 1 . Benchmarking with a cold plasma. Journal of Geophysical Research - Space Physics, 124 (11). pp. 88938912. ISSN 0148-0227 doi:

https://doi.org/10.1029/2019JA027088 Available at https://centaur.reading.ac.uk/86158/

It is advisable to refer to the publisher's version if you intend to cite from the work. See Guidance on citing.

To link to this article DOI: http://dx.doi.org/10.1029/2019JA027088

Publisher: American Geophysical Union

All outputs in CentAUR are protected by Intellectual Property Rights law, including copyright law. Copyright and IPR is retained by the creators or other copyright holders. Terms and conditions for use of this material are defined in the End User Agreement. 


\section{CentAUR}

Central Archive at the University of Reading

Reading's research outputs online 


\title{
Particle-in-cell experiments examine electron diffusion by whistler-mode waves: 1 . Benchmarking with a cold plasma
}

\author{
O. Allanson ${ }^{1}$, C. E. J. Watt ${ }^{1}$, H. Ratcliffe ${ }^{2}$, N. P. Meredith $^{3}$, H. J. Allison ${ }^{3,4}$, S. N. Bentley ${ }^{1}$, T. \\ Bloch $^{1} \&$ S. A. Glauert ${ }^{3}$

\begin{abstract}
${ }^{1}$ Space \& Atmospheric Electricity Group; Department of Meteorology; University of Reading; Reading; UK ${ }^{2}$ Centre for Fusion, Space and Astrophysics; Department of Physics; University of Warwick; Coventry; UK ${ }^{3}$ British Antarctic Survey, Natural Environment Research Council, Cambridge; UK

${ }^{4}$ Helmholtz Centre Potsdam - GFZ German Research Centre for Geosciences: Potsdam; Germany
\end{abstract}

\section{Key Points:}

- Particle-in-cell numerical experiments track electron pitch-angle diffusion due to whistler-mode wave-particle interactions

- Our novel approach directly extracts diffusive characteristics across all energy and pitch angle space

- After an initial transient phase we observe a normal diffusive response that is consistent with quasilinear theory 


\begin{abstract}
Using a particle-in-cell code, we study the diffusive response of electrons due to waveparticle interactions with whistler-mode waves. The relatively simple configuration of field-aligned waves in a cold plasma is used in order to benchmark our novel method, and to compare with previous works that used a different modelling technique. In this boundary-value problem, incoherent whistler-mode waves are excited at the domain boundary, and then propagate through the ambient plasma. Electron diffusion characteristics are directly extracted from particle data across all available energy and pitch-angle space. The 'nature' of the diffusive response is itself a function of energy and pitch-angle, such that the rate of diffusion is not always constant in time. However, after an initial transient phase, the rate of diffusion tends to a constant, in a manner that is consistent with the assumptions of quasilinear diffusion theory. This work establishes a framework for future investigations on the nature of diffusion due to whistler-mode wave-particle interactions, using particle-in-cell numerical codes with driven waves as boundary value problems.
\end{abstract}

\title{
Plain language summary
}

'Whistler-mode' plasma waves interact with electrons in the Earth's outer radiation belts. This wave-particle interaction plays a significant role in both electron acceleration, and in the loss of electrons to the atmosphere via 'pitch angle scattering'. Such processes are typically modelled using numerical diffusion codes, with electron diffusion coefficients that characterize the nature and the strength of the wave-particle interaction. These diffusion coefficients are calculated using a mixture of long-established theory and input parameters taken from data and/or empirical models. We present a novel method for the direct extraction of characteristics of the electron diffusion from particle-in-cell numerical experiments. Our results demonstrate that the rate of diffusion can be time-dependent at early times, but then tends to constant values in a manner that is consistent with quasilinear theory.

\section{Introduction}

Wave-particle interactions are a key source of variability in the outer radiation belt (e.g. Horne et al. [2005a]; Thorne [2010]; Reeves et al. [2013]). Decades of research into the behaviour of high-energy electrons in Earth's magnetosphere has determined that waveparticle interactions over a range of different frequencies can diffuse the particles in phasespace, leading to energisation and loss of high-energy particles that could explain the variability of the belts (e.g. Fälthammar [1965]; Hudson et al. [2000]; Thorne et al. [2013]). Whistler-mode waves are electromagnetic waves that propagate below the electron gyrofrequency (e.g. see Artemyev et al. [2016a]) and can interact with electrons across a wide range of energies (e.g. Horne et al. [2005b]; Thorne et al. [2010]). Whistler-mode waves take a range of different forms: narrowband transmitter waves are artificially-generated at Earth's surface by high-power radio transmitters (e.g. Zhang et al. [2018b]; Meredith et al. [2019]); lightning-generated whistlers are generated by lightning and propagate upwards through the ionosphere into the magnetosphere (e.g. Němec et al. [2010]); whistler-mode chorus is naturally generated by plasma instabilities within the Earth's magnetosphere (e.g. Omura et al. [2007]; Meredith et al. [2009]; Chen et al. [2017]; Gao et al. [2017]); incoherent plasmaspheric hiss [Bortnik et al., 2008a; Chen et al., 2012, 2014] has a number of established source mechanisms (e.g. see Meredith et al. [2018] for a discussion of these).

Current approaches to modelling the effect of wave-particle interactions in the outer radiation belt most typically use the quasilinear theory (QLT) [Kennel and Engelmann, 1966; Lerche, 1968; Lyons, 1974]. This formalism describes wave-particle interactions as diffusive processes in the plasma, flattening out gradients and moving electrons to different energies and/or different pitch-angles. The exact form of the equations used in the model depends on the characterization of the wave-particle interactions that are responsible for 
the diffusive behaviour, and this characterization is provided by the diffusion coefficients. The analytic form of diffusion coefficients are derived using QLT, and then implemented in diffusion codes by using models of wave and plasma parameters (e.g. see Glauert et al. [2014]). Formally, the application of QLT to wave-particle interactions places a number of restrictions on the plasma waves considered, typically considered to be that the waves are incoherent and of low amplitude [Stix, 1992; Treumann and Baumjohann, 2001]. However, observations of whistler-mode waves in the inner magnetosphere (e.g. Cattell et al. [2008]; Cully et al. [2008]; Breneman et al. [2011]; Wilson III et al. [2011]; Kellogg et al. [2011]; Gao et al. [2016]) have revealed that wave amplitudes can be orders of magnitude larger than previously thought. Furthermore, it is evident from observations that certain whistler-modes possess structure and/or coherency in frequency space, e.g. the rising and falling tones of chorus emissions, and the nearly monochromatic signals of transmitter waves. Hence there is strong evidence that motivates from-first-principles investigations of whistler-mode wave-particle interactions in the outer radiation belt.

There are a large number of theoretical calculations and numerical experiments relevant to the work presented in this paper and so it is not possible to discuss every one (e.g. a non-exhaustive list of such works on whistler-mode wave-particle interactions includes Albert [2001, 2002]; Omura et al. [2007]; Bortnik et al. [2008b]; Albert [2010]; Tao and Bortnik [2010]; Tao et al. [2011, 2012a,b, 2013]; Camporeale and Zimbardo [2015]; Camporeale [2015]; Mourenas et al. [2018]; Silva et al. [2018]). Instead, we focus on some of the works that - either with test-particle or particle-in-cell (PiC) codes - analyzed the statistical/diffusive response of the plasma, by directly extracting particle data. Bortnik et al. [2008a] used test-particle experiments in a dipolar magnetic field to model the effect of large amplitude and oblique monochromatic chorus waves on the particle response. It was found that the wave-particle interaction changed qualitatively from that of diffusion beyond a certain amplitude, in which case a nonlinear approach was found necessary. The nature of the nonlinear behaviour observed (diffusive, phase bunching or phase-trapping) was found to correlate with those predicted in Albert [2002] for different wave and plasma regimes. The nonlinear behaviour also varies according to a inhomogeneity parameter that indicates whether or not quasi-linear theory is applicable in the narrowband limit (discussed in e.g. Bortnik et al. [2008a]; Omura et al.; Tao and Bortnik [2010]; Tao et al. [2012a]). Tao et al. [2011] used a test-particle code to study the response of electrons to a uniform spectrum of incoherent, broadband and small amplitude waves in a homogeneous background field, but specifically targeted electron populations predicted to be in resonance. They found that the electron response was indeed stochastic and in excellent agreement with QLT. Tao et al. [2012a] performed test-particle simulations for field-aligned waves in a simplified dipole field model (no curvature), and found (also for resonant particles only) that the bounce-averaged quasi-linear diffusion coefficients became invalid as the wave amplitude surpassed given thresholds. Specifically, they found this threshold to be $\left|B_{\mathrm{w}, \mathrm{rms}}^{2} / B_{0}^{2}\right| \geq 2 \times 10^{-7}$ for $10 \mathrm{keV}$ electrons, and $\left|B_{\mathrm{w}, \mathrm{rms}}^{2} / B_{0}^{2}\right| \geq 7 \times 10^{-6}$ for $1 \mathrm{MeV}$ electrons, where waves have root-mean-squared amplitudes of magnitude $B_{\mathrm{w}, \mathrm{rms}}$ in a background field $B_{0}$. Camporeale and Zimbardo [2015] used self-consistent kinetic simulations to investigate diffusion during the linear growth phase and saturation of anisotropy-driven instabilities that self-consistently generate whistler-mode waves. They found evidence of nonlinear and time-dependent effects, with enhanced pitch angle diffusion during the linear growth phase. In a similar experiment, Camporeale [2015] investigated diffusion due to the self-consistently generated lower-band chorus waves, and compared to the predictions given by a QLT diffusion code. Specifically, they found significant mismatch in regions of phase-space for which the resonance condition is not satisfied, and called for nonlinear theories in order to capture non-resonant interactions. We also note that there is recent theoretical work based upon using kinetic equations used to describe the evolution of the particle energy distribution due to nonlinear wave-particle interactions Artemyev et al. [2016b, 2017, 2018]; Mourenas et al. [2018]; Vainchtein et al. [2018], and for which one of the main aims is "to incorporate nonlinear effects of intense, short-duration chorus wave packets into global (quasilinear) diffusion models" (Quoted from presentation, J. Bortnik, 
ISSS-13, September 6-14 2018, UCLA). We note that the standard quasilinear diffusion theory sometimes captures observed diffusive properties surprisingly well, even in circumstances for which the assumptions of the theory are formally invalid (e.g. see a discussion in Zhang et al. [2018a]).

The standard test-particle approach to modelling wave-particle interactions presents both advantages and disadvantages. Test-particle codes are (relatively) cheap to run numerically and enable one to implement wave modes of exactly the desired form. However, wave-particle interactions are a fundamentally kinetic physics process, and test-particle codes do not include all of the self-consistent interactions between particles and wave fields. Particle-in-cell experiments enable users to model these self-consistent interactions, and in principle allow a greater range of kinetic-physics diagnostics (e.g. the distribution function). Indeed, it has recently been shown that using the two different approaches to study diffusion due to whistler mode wave-particle interactions can yield markedly different results [Camporeale and Zimbardo, 2015; Camporeale, 2015]. Here, we use a mixture of both the test-particle and particle-in-cell approaches. We exploit the self-consistent $\mathrm{PiC}$ interaction to model the waves as they propagate through the plasma, instead of prescribing fixed-characteristic waves as in a test-particle approach. The inclusion of sub electron-scale physics in the interaction between the background plasma and the propagating waves will allow the whistler-mode waves to fluctuate on sub-electron spatial and temporal scales. In the simulation, we release a very large number of tracer ('test') particles in order to extract characteristics of the pitch-angle diffusion for electrons across all available energies and pitch-angles. These tracer particles contribute no 'moments' to the particle-in-cell algorithm, and respond to the electromagnetic fields in the same way as the methods in Tao et al. [2011], for example. In using a combination of both self-consistent particle-in-cell and test-particle methods, we should expect to see both similarities and differences in the results obtained when compared to Tao et al. [2011].

In particular, our intent in this first paper of a series is to study the nature of the diffusion when the waves propagate in one dimension along the background magnetic field in a cold, homogeneous plasma. This initial 'benchmarking' scenario showcases our method and indicates similarities to and differences from the test-particle results reported in previous work (e.g. Tao et al. [2011]). Future papers in this series will compare the strength of the effective diffusion coefficients extracted from the PiC experiment with the size of the analytic quasilinear diffusion coefficient across all of pitch-angle/energy space, and will repeat the numerical experiment for whistler-mode wave propagation through a plasma with the fractional warm components that can be found in Earth's inner magnetosphere.

This paper is organized as follows. In Section 2 we describe the philosophy and setup of the numerical experiments, including the numerical scheme. In particular we discuss the wave excitation mechanism and the properties of the electromagnetic waves within the domain. Diffusion theory as is applicable to the outer radiation belt is discussed in Section 3. Results from the numerical experiments, including the diffusive plasma response are discussed in Section 4. In Section 5 we discuss our results in more detail and put the results in context. Section 6 contains a summary, including motivation for future investigations that will build upon the results reported here.

\section{Outline of experiments}

Radiation belt diffusion coefficients are fundamentally a function of both the plasma and wave parameters, i.e,. the plasma density, background magnetic field, wave strength and wave spectral form. As a result, the direct evaluation of a diffusion coefficient relies on both the plasma and wave characteristics being quasi-static for the time over which they are calculated [Schulz and Lanzerotti, 1974]. Therefore we consider a boundary value problem, in which we perturb the left-hand boundary with a given specific wave spectrum, which then excites electromagnetic waves that propagate throughout the experimental do- 
main. The perturbation mechanism is applied at all times, and this enables us to study the interaction for a wave spectrum that is quasi-static in amplitude (root-mean-squared) and spectral form. However, the wave spectrum does exhibit some small-scale spatial and temporal fluctuations in response to self-consistent interactions with the background cold plasma. This approach is in contrast to an initial value problem, in which one might study the self-consistent generation mechanism and subsequent evolution of waves in an initially unstable plasma, and for which the wave spectra is more variable in time (e.g. Katoh and Omura [2006]; Omura et al.; Hikishima et al. [2009]; Omura et al. [2009]; Omidi et al. [2010, 2011]; Katoh and Omura [2013]; Camporeale [2015]; Camporeale and Zimbardo [2015]; Silva et al. [2017]; Ratcliffe and Watt [2017]; Katoh et al. [2018]). In Tao et al. [2011], a relativistic test-particle code was used to study the diffusive plasma response due to wave-particle interactions of driven, broadband and incoherent waves waves with magnetospheric plasma populations appropriate for the outer radiation belt at 6 Earth radii $\left(r \sim 6 R_{\mathrm{E}}\right)$. Our experimental parameters are chosen in order to resemble those in Tao et al. [2011] as far as possible.

\subsection{Numerical experiment design}

Wave-particle interactions are a fundamentally kinetic plasma physics process, since their efficiency explicitly depends on particle-scale physics, as statistically described by the particle distribution function. We use the EPOCH PiC code [Arber et al., 2015], which is described in more detail in Appendix A. Essentially, the numerical experiments involve exciting waves of specified frequencies from an "antenna" at one of the spatial boundaries of the simulation domain. Electromagnetic waves then propagate through the domain, selfconsistently interacting with the cold plasma component of the plasma interior. We also release a large number of non-interacting 'tracer' particles that are used to monitor the diffusion in different regions of pitch-angle/energy space. These particles contribute no moments to the $\mathrm{PiC}$ algorithm. The tracer particles act as 'labels' in phase-space such that their collective diffusive response can be categorized as a function of pitch angle and energy.

As a first step, we restrict the experimental domain to one field-aligned dimension $(x)$. On the compressed dayside magnetosphere near the magnetic equator, uniform fields and field-aligned wave propagation is a reasonable approximation [Tsurutani and Smith, 1977]. We do so in order to fully understand the most idealized example of this waveparticle interaction, before introducing new effects in isolation (e.g. oblique wave propagation, field inhomogeneities, and modifications to the wave spectra). EPOCH uses a Cartesian grid, which in 1-D means that all quantities may vary in the $x$ direction only. We set an ambient magnetic field, $\mathbf{B}_{0}=\left(B_{x 0}, 0,0\right)$, with $B_{x 0}=140 \mathrm{nT}$, and so refer to $x$ as parallel $(\|), y$ and $z$ as perpendicular $(\perp)$.

We model a uniform cold 'background' plasma with number density, $n_{\mathrm{b}}=10^{7} \mathrm{~m}^{-3}$. For this cold background plasma, and ambient magnetic field, the ratio of electron plasma frequency to non-relativistic gyrofrequency is given by $\omega_{\mathrm{pe}} /\left|\omega_{\mathrm{ce}}\right| \approx 7.2$, for $\omega_{\mathrm{pe}}=\sqrt{n e^{2} /\left(m_{e} \epsilon_{0}\right)}$, and $\omega_{\mathrm{ce}}=q_{e} B_{0} / m_{e}<0$ in the uniform background field. We describe the ambient plasma by using cold ion and electron populations with initial spatially uniform number density of $n_{\mathrm{b}}$ and isotropic temperature of $0.1 \mathrm{eV}$. These choices are motivated by our desire to emulate, as far as possible, the scenario of Tao et al. [2011]. In a warm plasma, or plasma with fractional warm components, the wave amplitudes would be expected to altered by the presence of the warm component. If the plasma is isotropic, then the waves will damp/reduce their amplitude, and if the plasma has positive anisotropy then the waves may grow in amplitude through the appropriate kinetic wave-particle interaction. In this numerical experiment, we choose a cold plasma environment in order to ensure that waves entering the domain do not change amplitude significantly as they propagate through the plasma. 
The real world run time for the experiment is $T=575 t_{\mathrm{ce}} \approx 0.15 \mathrm{~s}$, for $t_{\mathrm{ce}}=1 / f_{\mathrm{ce}}$ with $f_{\mathrm{ce}}=\left|\omega_{\mathrm{ce}}\right| /(2 \pi) \approx 3919 \mathrm{~Hz}$. We use 500 particles-per-cell per species; physical values of proton-electron mass ratio, $m_{\mathrm{i}} / m_{\mathrm{e}}=1836.2$; and the speed of light is set to its real value $c \approx 3 \times 10^{8} \mathrm{~ms}^{-1}$. Periodic boundary conditions are chosen for the particles, whereas electromagnetic waves have open boundary conditions (the electromagnetic field boundary condition works by allowing outflowing characteristics to propagate through the boundary with as little reflection as possible). The domain length, $L=40 \lambda_{\mathrm{lc}}$, is set to be 40 times the estimated wavelength inside the domain, $\lambda_{\mathrm{lc}}=c /\left(\eta f_{\mathrm{lc}}\right)$, of the lowest frequency wave emitted by the antenna, $\omega_{\mathrm{lc}}=2 \pi f_{\mathrm{lc}}$. The refractive index is a function of frequency, $\eta=\eta(\omega)$, and is determined by the cold plasma dispersion relation [Stix, 1992; Omura et al., 2007],

$$
\eta^{2}=\frac{c^{2} k^{2}}{\omega^{2}}=1+\frac{\omega_{\mathrm{pe}}^{2} / \omega^{2}}{\left|\omega_{\mathrm{ce}}\right| / \omega-1} .
$$

We use 3587 cells in the $x$-direction, with grid spacing $\Delta x \approx 235 \mathrm{~m}$, such that $\Delta x /\left(c /\left|\omega_{\text {ce }}\right|\right) \approx$ 0.02 . The Debye radius, $\lambda_{D}=\sqrt{\epsilon_{0} k_{B} T_{e} /\left(n_{b} q_{e}^{2}\right)} \approx 2.35 \mathrm{~m}$ is not resolved by $\Delta x$, and we have $\Delta x / \lambda_{D}=100$. It is well-known that particle-in-cell experiments are in principle vulnerable to the self-heating phenomenon, and that this can be mitigated by choosing $\Delta x \approx \lambda_{D}$ in explicit codes. However, it is absolutely possible to perform valid and physically meaningful particle-in-cell numerical experiments with a value of $\Delta x$ that does not resolve the Debye radius (e.g. see a discussion in [Arber et al., 2015]). In particular, if one can demonstrate that for a particular choice of $\Delta x$ :

1. all necessary physical scales have been resolved that are most important for the phenomenon of interest,

2. the self-heating is limited to a reasonable level,

then it is entirely justifiable to have $\Delta x>\lambda_{D}$. It is not uncommon to use such values, e.g. two examples of works that discussed results of explicit PiC experiments using a spatial resolution $\Delta \gg \lambda_{D}$ include: (i) Ratcliffe and Watt [2017] with $\Delta / \lambda_{D} \approx 139$, in which the ' 0.5 cyclotron frequency gap' in magnetospheric whistler-mode waves was selfconsistently generated; (ii) Tsiklauri [2016] with $\Delta / \lambda_{D} \approx 200-270$, for the study of electron plasma wakefield acceleration.

We justify point 1 . for our experiment as follows. Our chosen value of $\Delta x$ resolves the electron inertial length according to $\Delta x / d_{e} \approx 0.07$, for $d_{e}=c / \omega_{\mathrm{pe}} \approx 1680 \mathrm{~m}$, and therefore resolves the fundamental scale of electron kinetic physics. Furthermore, it resolves the shortest wavelength of the whistler-mode wave spectrum that is important for the wave-particle dynamics in this study, $\Delta x / \lambda_{\mathrm{uc}} \approx 0.009$, for $\lambda_{\mathrm{uc}}=c /\left(\eta f_{\mathrm{uc}}\right)$ and $\eta$ determined by equation (1). Therefore, our chosen grid discretization resolves electron scale kinetic physics, and in particular the spatial scales necessary for electron wave-particle interactions with the driven whistler-mode waves.

We justify point 2 . for our experiment as follows. The classic constraint $\Delta x \approx \lambda_{D}$ [Langdon, 1970] relates to the case where particle forces are assigned to 'nearest-neighbour' grid points, and for which the underlying scheme is momentum-conserving. EPOCH is a charge-conserving code, with capability to use higher-order shape functions: such weighting schemes suffer from a less catastrophic form of self-heating, and generally with a low growth rate. In this work we triangular shape functions (a 3 point stencil, 2 cells wide). A discussion of stability and self-heating in $\mathrm{EPOCH}$, with reference to shape-functions, is given in Section 5 of Arber et al. [2015]. The EPOCH code also provides one further method to limit the noise in the PiC simulation, and which we use in this work: ' $\delta F$ mode' (as detailed in Appendix A). In figure 1 we present an analysis of the temporal evolution of the electromagnetic and particle energies within the experimental domain, with special consideration of the electromagnetic energy flux through the boundaries. The experiment utilizes periodic boundary conditions for the particles. However, there is a constant input of electromagnetic energy into the domain via the wave excitation method, 

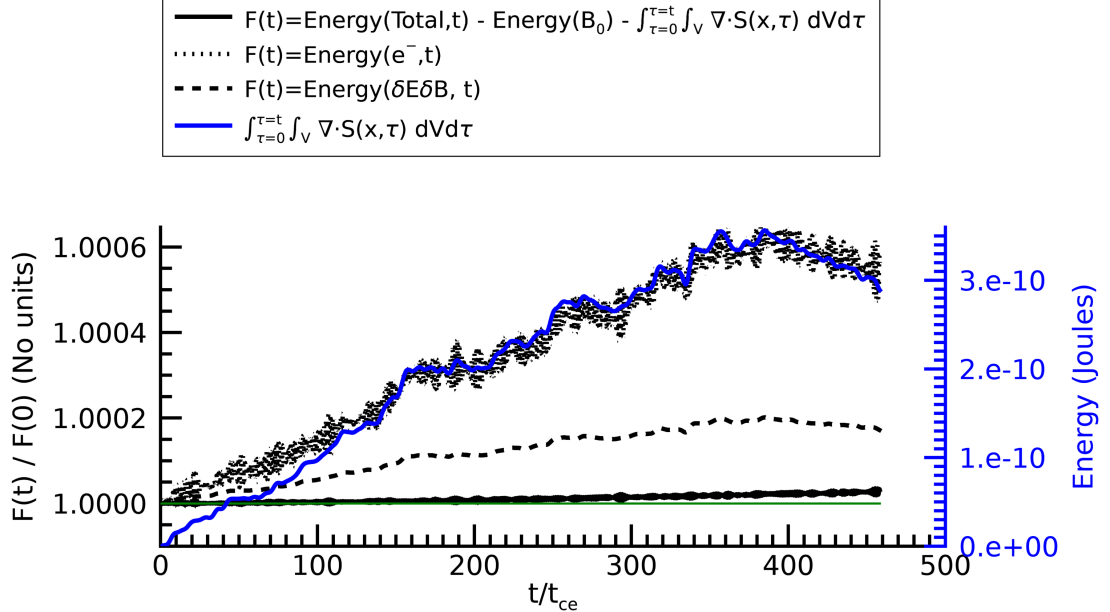

Figure 1. Evolution of the energy budget within the experiment.

and outgoing electromagnetic wave energy is permitted to flow out of the experimental domain. Therefore the total energy will not be conserved, but the following quantity should be conserved,

$$
E_{K E}(t)+E_{E M, \text { domain }}(t)-E_{S, \text { boundary }}(t)=\text { const. }
$$

The three terms on the left-hand-side represent, respectively: (a) the kinetic energy of all ions and electrons; (b) the total electromagnetic energy within the domain; (c) the timeintegrated net electromagnetic power that has entered the domain up to time $t$,

$$
E_{S, \text { boundary }}(t):=\int_{\tau=0}^{\tau=t} \int_{V} \nabla \cdot \mathbf{S}(x, t) d V d \tau
$$

for $\mathbf{S}$ the Poynting vector. This time-integrated power (energy) represents the only means by which the total energy budget within the experimental domain should change. In figure 1 we plot the normalized evolution of the quantity in equation (2) minus the energy associated with the time-independent and homogeneous background magnetic field (with a solid black line). Stability of the numerical code required that this quantity should not diverge significantly from unity. Figure 1 shows conservation of the net simulation energy to better than $0.1 \%$, confirming that self heating is minimal. In figure 1 we also plot the evolution of: total electron energy (black dots); total energy associated with electromagnetic fluctuations within the domain (black dashes); and the time-integrated electromagnetic power that has entered the domain up to time $t$ (solid blue line). We see that the total electron energy clearly tracks the injected wave energy and the total energy associated with the EM fluctuations.

\subsection{Wave spectra}

We excite the plasma at the left-hand boundary $(x=0)$ using EPOCH's using EPOCH's driven boundary option, (called 'laser' after its most common use), such that the perturbations propagate in the positive- $x$ direction. For each given specified frequency, this boundary condition simply perturbs the domain with a (sinusoidal) time-varying electromagnetic field. We superpose a collection of different perturbations, with the intent to reproduce the spectrum used in Tao et al. [2011]. We specify a discrete sum of $N_{\text {wave }}=$ 100 individual right-hand polarized electric field perturbations. Each one of the 100 modes 
is composed of 2 linearly polarized components, with wave fields that oscillate in the $y$ and $z$ directions respectively,

$$
\mathbf{E}_{\text {wave }}(x, t)=\sum_{i=1}^{N_{\text {wave }}} \mathbf{E}_{\text {wave }, i}(x, t)=\sum_{i=1}^{N_{\text {wave }}} \mathbf{E}_{\text {wave }, y, i}(x, t)+\mathbf{E}_{\text {wave }, z, i}(x, t),
$$

Each pair of linearly polarized waves, $\left(\mathbf{E}_{\mathrm{wave}, y, i}, \mathbf{E}_{\mathrm{wave}, z, i}\right)$, has the appropriate phase shift between themselves such as is required for right-handed polarization [Stix, 1992]. Each mode, $\mathbf{E}_{\mathrm{wave}, i}$, also has a random phase, and a frequency that is uniformly selected from the range

$$
f_{\mathrm{lc}}=0.2 f_{\mathrm{ce}} \leq f \leq 0.4 f_{\mathrm{ce}}=f_{\mathrm{uc}}
$$

Full details on how to prescribe such a spectrum are given in Tao et al. [2011], and we include the text file that prescribes the perturbation as supplementary information (S2).

Given these user inputs for the electric field perturbations, the Maxwell solver in EPOCH generates self-consistent magnetic field perturbations $\left(\mathbf{B}_{\text {wave }, i}\right)$ in accordance with Maxwell's equations. We choose the magnitude of the electric field perturbations so that they are (in principle) consistent with corresponding magnetic field perturbations of $1 \mathrm{pT}$ in a vacuum $\left(\left|\mathbf{E}_{\text {wave }, i}\right|=c\left|\mathbf{B}_{\text {wave }, i}\right|\right.$, and such that $\left.\left|\mathbf{B}_{\text {wave }, i}\right|=1 \mathrm{pT}\right)$. We note here that our perturbation method is intended to excite the whistler-mode wave branch of the cold plasma dispersion relation [Stix, 1992], since a cold plasma can support whistler-mode wave propagation when excited at these frequencies. A 'fully self-consistent' wave driving technique would also necessitate the self-consistent perturbations of other oscillating macroscopic quantities, e.g. polarization currents. However, that approach is much more complicated and beyond the scope of this work. Regardless of the specific excitation technique, and as will be shown, we are able to excite the electromagnetic component of whistler-mode waves within the interior of the plasma. Once excited, these components then propagate and continue to be supported by (and interact self-consistently with) the background cold-plasma.

Sudden electromagnetic perturbations can often cause undesired 'shock' effects in a simulated plasma at the moment the perturbation is 'switched on'. In order to eliminate any such effects, we apply a linear envelope to the wave profile, so that for $t<2 / f_{\mathrm{lc}}$, the wave profile has amplitude scaled by $1 / t$. This prevents any shock effects from occurring. It takes approximately $t_{\text {cross }}=115 t_{c e}$ for the wave profile to cross the experimental domain, from left to right. All wave and particle analysis in this paper pertains to times after this time, $t>t_{\text {cross }}$. Therefore we analyze only over times during which the entire experimental domain is interacting with the propagating waves. Since the run time of the experiment is $T=575 t_{c e}$, we analyze wave-particle interactions for a total time of $T-t_{\text {cross }}=460 t_{c e}$. This corresponds to $\approx 92$ wave periods for waves with frequency $f_{l c}$, and $\approx 184$ wave periods for waves with frequency $f_{u c}$. For completeness, we plot the $B_{y}$ (red) and $E_{y}$ (blue) components of the electromagnetic fields as a function of $x$ in figure 2. This figure includes the waveform at: (a) $28 t_{\text {ce }}$, soon after the completion of the linear envelope scaling of the excitation; (b) $143 t_{\mathrm{ce}}$, soon after the wave has crossed the domain; (c) $280 t_{\mathrm{ce}}$, roughly half-way through the numerical experiment; (d) $561 t_{\mathrm{ce}}$, close to the end of the experiment.

One important difference between our work and that of Tao et al. [2011], is that the wave-spectrum excited by the antenna on the left-hand boundary is not exactly reproduced within the domain. Since we use a fully kinetic numerical code, the driven waves interact self-consistently with the background plasma populations via Maxwell's equations, where the current density is derived directly from the flux of different species' super-particle populations. The background plasma super-particle populations evolve in a self-consistent manner via the (relativistic) Lorentz force equation. This is all at the cost of higher computational expense, as compared to a test-particle simulation. However, we consider the freedom to have fully self-consistent wave-particle interactions to be a benefit of using the 
(a)

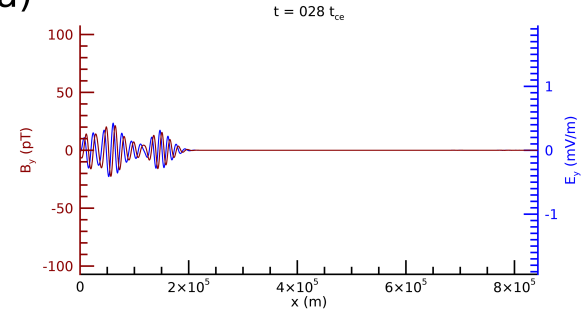

(c)

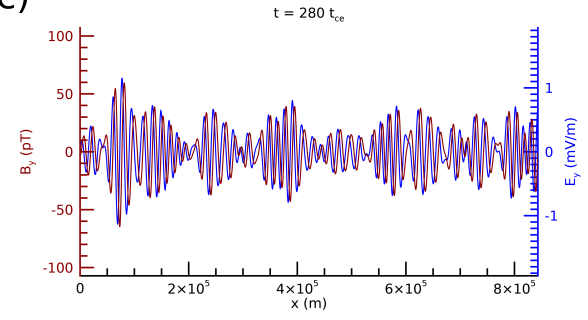

(b)

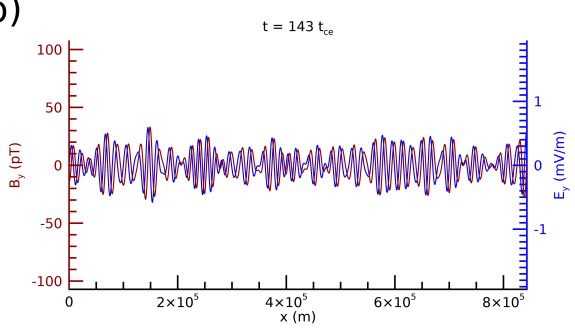

(d)

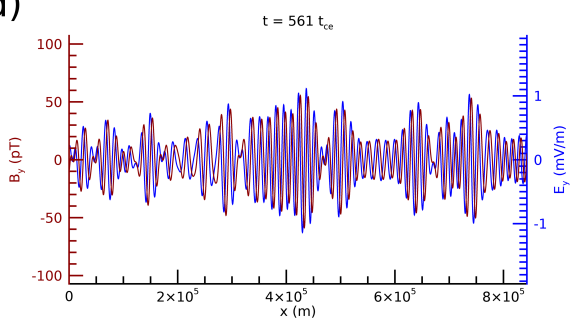

Figure 2. The $B_{y}$ (red) and $E_{y}$ (blue) components of the electromagnetic fields as a function of $x$ at: (a) $28 t_{\mathrm{ce}}$; (b) $143 t_{\mathrm{ce}}$; (c) $280 t_{\mathrm{ce}}$; (d) $561 t_{\mathrm{ce}}$

$\mathrm{PiC}$ approach for this study, and therefore the benefits of using fully self-consistent $\mathrm{PiC}$ compensate for the added computational cost.

Figure 3 shows the 'one-sided' Fourier amplitude spectrum of the $B_{y}$ (red) and $E_{y}$ components (blue) of the waves, averaged over all space (within the PiC domain) and time during the wave-particle interaction $\left(460 t_{c e}\right.$, i.e. $\left.t>t_{\text {cross }}\right)$. The Fourier amplitude is defined such that a single wave $\sim A \sin (k x \pm w t)$ will have amplitude $|A|$. The vertical black lines mark the lower and upper bounds of the driven wave spectrum $\left(f_{l c}=0.2 f_{c e}\right.$ and $f_{u c}=0.4 f_{c e}$ respectively), whilst the vertical green line marks the electron gyrofrequency $f_{c e}$. The horizontal black line marks a continuous version of the $B_{y}$ spectrum employed by Tao et al. [2011]. The waves clearly show dominant power within the required frequency domain. However, there is some amplification as compared to the uniform 1pT spectrum as used by Tao et al. [2011], and we observe non-zero amplitudes outside the driven frequency domain $\left(f_{l c}, f_{u c}\right)$. We observe a root-mean-square wave amplitude of $B_{\mathrm{w}, \mathrm{rms}} \approx 25 \mathrm{pT}$ in our experiment, slightly higher than the value of $10 \mathrm{pT}$ that Tao et al. [2011] use. This difference is, in reality, a small one, and a result of the difficulty in exactly prescribing a given wave spectrum in a $\mathrm{PiC}$ experiment. This amplitude $\left(\left(B_{\mathrm{w}, \mathrm{rms}} / B_{0}\right)^{2} \approx 3 \times 10^{-8}\right)$ still falls well below the nonlinear wave amplitude thresholds as discussed in Section 1. Therefore, this factor does not preclude us from comparing our results to those obtained by Tao et al. [2011]. As discussed above, we excite the boundary with individual whistler modes each with electric field amplitudes that correspond to magnetic field perturbations of $1 p T$, but for the case of a vacuum $(|\delta \mathbf{B}| \approx|\delta \mathbf{E}| / c)$. However, when propagating through a plasma medium, one will expect magnetic field perturbations within the domain $|\delta \mathbf{B}| \approx|\delta \mathbf{E}| /(c / \eta)$ (in our experiment $14.8<\eta<18.1$ for waves with frequencies $f_{\mathrm{lc}}<f<f_{\mathrm{uc}}$ ). If the coupling efficiency between the wave excitation mechanism and the plasma was $100 \%$ efficient, then we should expect a spectrum of magnetic field perturbations (i.e. at each frequency) with amplitudes a factor of $\eta$ greater than $|\delta \mathbf{E}| / c$, i.e $\approx \eta \times 1 \mathrm{pT}$. However, the coupling is not perfectly efficient, as should be expected, and so we observe magnetic field perturbations within the domain $\approx 2-3$ times the 1pT level used by Tao et al. [2011]. One obtains near-identical results as those in figure 3 for the power spectra of the $B_{z}$ and $E_{z}$ components, as should be expected for circularly polarized waves. 


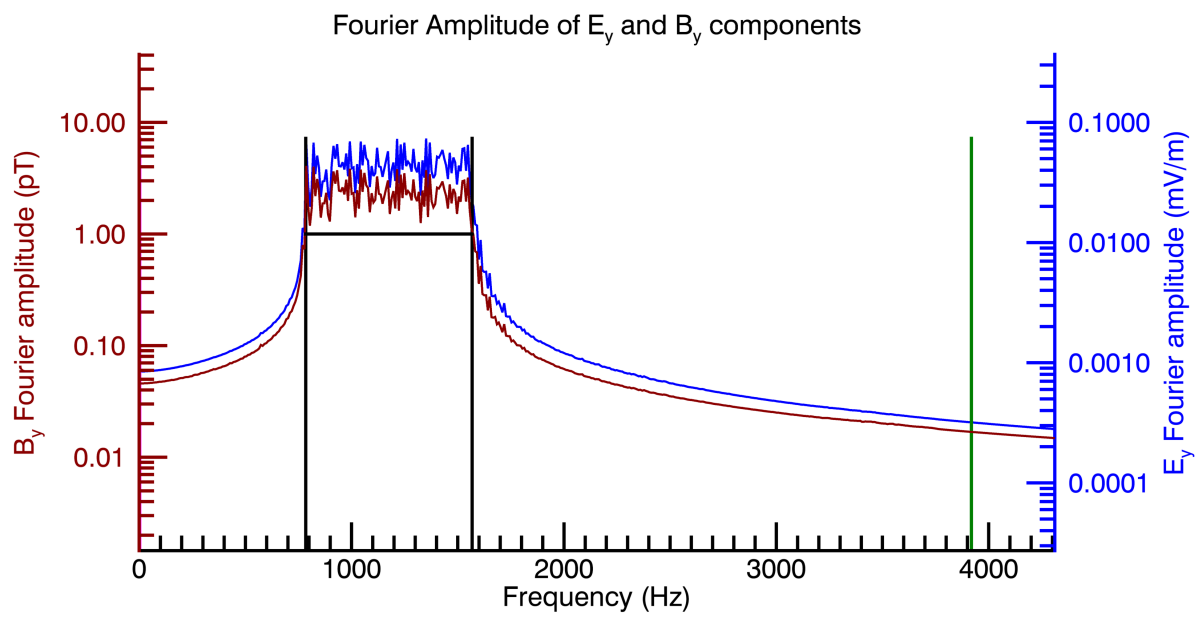

Figure 3. Fourier amplitude of the $B_{y}$ (red) and $E_{y}$ components (blue) of the waves within the PiC domain. Vertical black lines mark the lower and upper bounds of the driven wave spectrum $\left(f_{l c}=0.2 f_{c e}\right.$ and $f_{u c}=0.4 f_{c e}$ respectively). Vertical green line marks the electron gyrofrequency $f_{c e}$. The horizontal black line marks a continuous version of the $B_{y}$ spectrum employed by Tao et al. [2011].

Figure 4 shows the 'dispersion relation' of the $B_{y}$ component of the waves present, obtained via Fourier transforms performed over the entire spatial and temporal domain during the wave-particle interaction. The over-plotted line marks the cold plasma dispersion relation as according to equation (1), and we see that the dominant power is strongly localized to the $\left(f_{l c}, f_{u c}\right)$ region, and along the expected dispersion curve. Once again, we obtain near-identical plots of the dispersion relation for $B_{z}, E_{y}$ and $E_{z}$.

\section{Particle diffusion}

The application of QLT to the Vlasov-Maxwell equation for collisionless plasmas leads to a diffusion equation to describe the plasma distribution function, $F$, of the form

$$
\frac{\partial F}{\partial t}=\sum_{i, j} \frac{\partial}{\partial J_{i}}\left[D_{i j} \frac{\partial F}{\partial J_{j}}\right]
$$

[Schulz and Lanzerotti, 1974], for $D_{i j}$ a symmetric tensor of diffusion coefficients, and $J_{i}$ are the three action integrals associated with adiabatic charged particle motion [Northrop, 1963; Roederer and Zhang, 2013]. For use in the outer radiation belt, equation (3) is typically rewritten in $\left(E, \alpha, L^{\star}\right)$ space (e.g. see Glauert et al. [2014]). Here, the energy $E=$ $p^{2} /\left(2 m_{0 e}\right)$ (for $\mathbf{p}=\gamma m_{0 e} \mathbf{v}$, with $\gamma$ the relativistic gamma, and $m_{0 e}$ the electron rest mass); pitch angle $\alpha=\tan ^{-1}\left(\left|p_{\perp} / p_{\|}\right|\right)$(for $p_{\perp}$ and $p_{\|}$the momenta perpendicular and parallel to the background magnetic field); and $L^{\star} \propto 1 / \Phi$, is a value inversely proportional to the third adiabatic invariant [Roederer and Zhang, 2013; Roederer and Lejosne, 2018]. The work in this paper does not consider radial diffusion (i.e. diffusion in $L^{\star}$ ). It is the diffusion in pitch angle, characterized by $D_{\alpha \alpha}=D_{\alpha \alpha}(E, \alpha)$, that will be the focus.

Particles are considered to be in resonance with a given wave mode when the waveparticle resonance condition is satisfied [Kennel and Engelmann, 1966],

$$
\omega-k_{\|} v_{\|}=n \omega_{\mathrm{ce}} / \gamma
$$




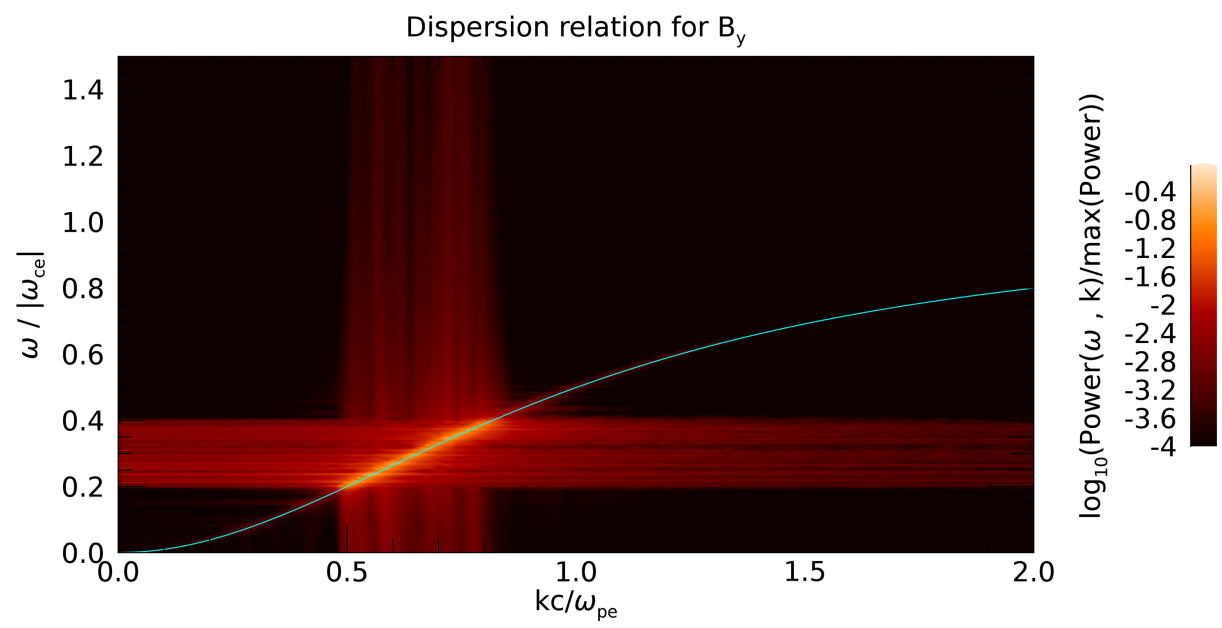

Figure 4. 'Dispersion relation' of the $B_{y}$ component of the waves present in the numerical experiment, averaged over the entire spatial and temporal domain during the wave-particle interaction. The over-plotted cyan curve marks the cold plasma dispersion relation (see equation (1)).

In this equation, $\omega>0$ is the wave frequency, $n=0, \pm 1, \pm 2, \ldots ; k_{\|}=\mathbf{k} \cdot \mathbf{B}_{0} / B_{0}$ and $v_{\|}=p_{\|} /\left(m_{0 e} \gamma\right)$ are the wave vector and velocity components that are parallel to $B_{0}$, and we remind that $\omega_{\mathrm{ce}}<0$. In the case of field-aligned whistler mode waves, only the $n=-1$ resonance can occur for electrons (e.g. see Summers [2005]). Under these circumstances, wave-particle resonance occurs for a given wave frequency, $\omega$, for pitch angles and energies defined by

$$
\alpha=\cos ^{-1}\left(\left|\frac{\mid \omega_{\mathrm{ce}} / \omega-(1+\varepsilon)}{(k c / \omega) \sqrt{\varepsilon^{2}+2 \varepsilon}}\right|\right),
$$

for $\varepsilon=E /\left(m_{0 e} c^{2}\right)$, and $k c / \omega$ given by equation (1). This equation implies that for a given pitch angle, lower frequency waves resonate with higher energies [Camporeale, 2015; Chen et al., 2018]. Furthermore, for a given wave frequency, the values of particle energy that can resonate are a monotonically increasing function of pitch angle.

\subsection{Background theory on normal diffusion}

An implicit assumption in the use of QLT is that the plasma undergoes 'normal diffusion' [Bouchaud and Georges, 1990] in phase-space: "the diffusion model assumes the existence of an underlying uncorrelated, Gaussian stochastic process, i.e., a Brownian random walk" [del Castillo-Negrete et al., 2004]. In this normal diffusive framework, diffusion coefficients are defined by the following formula (for arbitrary variables $X$ and $Y$ ),

$$
D_{X Y}(X, Y)=\frac{\left\langle\Delta X_{l}\right\rangle\left\langle\Delta Y_{l}\right\rangle}{2 \Delta t},
$$

in units of $[X][Y] s^{-1}$. Here, $\Delta t$ is interpreted as the duration over which the diffusion coefficients are to be calculated, $X_{l}$ and $Y_{l}$ are distributions of the particle parameters, localized to given values of $X$ and $Y$ (for $N$ particles $l=1,2, \ldots, N$ within the given local population). The mean of the distribution $X_{l}$ is denoted by $\left\langle X_{l}\right\rangle$, and

$$
\left\langle\Delta X_{l}\right\rangle \equiv\left\langle X_{l}-\left\langle X_{l}\right\rangle\right\rangle,
$$

such that $\left\langle\Delta X_{l}\right\rangle^{2} \equiv \operatorname{var}\left(X_{l}\right)$, for $\operatorname{var}\left(X_{l}\right)$ the variance of the distribution $X_{l}$. Implicit within this normal diffusion construction, is that the variance of a given parameter scales linearly 
with time,

$$
\Delta \operatorname{var}\left(X_{l}\right)=2 D_{X X} \Delta t,
$$

for $\Delta \operatorname{var}\left(X_{l}\right)$ the change in the variance of $X_{l}$ over the time $\Delta t$ (e.g. see Bouchaud and Georges [1990]; Metzler and Klafter [2000]). Conceptually, this means that for a given subset of a plasma population that is located in some region of phase space, then the distribution of that subset will spread in phase space according to equation (8).

\section{Diffusion in our numerical experiments}

Particle diffusion due to wave-particle interactions is monitored by using the EPOCH 'tracer' particle feature. EPOCH allows the embedding of test-particle populations (tracers) into numerical experiment. Unlike all the other particle populations, tracer particles do not contribute to the current, and so they effectively act as labels in phase-space: that is to say that the behaviour of a given tracer is indicative of the behaviour of an interacting particle (one that does contribute moments) in the same given region of phase-space. We release $\approx 10^{8}$ tracer particles within the domain at $t=0$, that are initially distributed according to a $100 \mathrm{keV}$ Maxwellian distribution that is uniform in space. Since they do not contribute current, we can load tracers however we want in phase space. We choose this specific 'temperature' merely to provide a relatively uniform distribution of particles across the section of energy space important for pitch-angle diffusion in the Radiation Belts.

Once the driven wave profile crosses the experimental domain, we consider all of the tracer particles to be under the influence of the whistler-mode waves. At $t=t_{\text {cross }}$, we bin the tracer particles in two dimensions according to their values of energy and pitch angle at that time. We emphasize that tracers then remain identified with that given bin for the entirety of the experiment, i.e. we do not re-bin at each data-dump. The binning process is performed as follows. We first order all of the $\approx 10^{8}$ tracers according to their energy at $t=t_{\text {cross }}$, and separate these tracers into 250 intervals, which are defined so as to allow exactly identical numbers of tracers in each interval. Within each of these energy intervals, the tracers are then ordered according to their value of pitch angle, and subdivided into 90 pitch angle sub-intervals, defined in order to allow the same number of tracers in each. Each of these $250 \times 90$ bins in energy and pitch-angle space contains 4444 tracer particles, and therefore we have uniformly good statistics within each bin with which to calculate the diffusive response. In the case of an isotropic Maxwellian distribution, it would be expected that this procedure would yield bins of a uniform size in pitch angle space, within each energy interval. Our bins are not exactly uniform in pitch angle space, but they are almost uniform. The reason is as follows. The tracer particles are loaded into the simulation at $t=0$ as an isotropic Maxwellian. However, they are binned at a later time, $t=t_{\text {cross }}$. Between $t=0$ and $t=t_{\text {cross }}$, the tracers have been responding to the electromagnetic perturbations within the domain created by both the wave excitation mechanism and any other inherent $\mathrm{PiC}$ electromagnetic fluctuations, and therefore the tracers are not be in a perfectly isotropic state at $t=t_{\text {cross }}$.

\subsection{Scattering in phase-space}

Figure 5 shows an example of the diffusive response for particles within a bin roughly centred on $\alpha=75^{\circ}$ and $E=50 \mathrm{keV}$. This combination of energy and pitch angle implies that the particles are in resonance with the driven wave spectrum. From hereon in we redefine $t=t_{\text {cross }}$ as $\tilde{t}=0$, and the end of the simulation as $\tilde{t}=T$, to simplify the discussion. Figures 5(a)-(d) plot electron $E, \alpha$ values at $\tilde{t}=0, T / 3,2 T / 3$ and $\tilde{t}=T$ respectively, and figures 5(e)-(h) plot pitch angle distributions of the entire sub-population (all energies). It is evident that pitch-angle diffusion dominates over energy diffusion in this bin, for reasoning as follows. The maximum magnitude of the pitch angle scattering in this bin reaches values of $(\max (\Delta \alpha))^{2} /(2 T) \approx( \pm 3)^{2} /(2 T)=9 /(2 T)$. Whereas the en- 
ergy scattering in this bin reaches maximum magnitudes of $\left(1 / E^{2}\right)(\max (\Delta E))^{2} /(2 T) \approx$ $(1 / 50 \mathrm{keV})^{2}( \pm 1 \mathrm{keV})^{2} /(2 T)=0.0004 /(2 T)$. We have checked that the dominance of pitchangle scattering is observed in most bins. This is an expected result, since the ratio $f_{\mathrm{pe}} / f_{\mathrm{ce}}$ is known to control the relative significance of energy versus pitch angle diffusion [Summers, 2005]. It is also interesting to note that the particles are scattered in preferred directions. This scattering is such that positive changes in $E$ correlate with positive changes in $\alpha$, and negative changes in $E$ correlate with negative changes in $\alpha$. We have observed that this behaviour is ubiquitous for the particles that are in resonance with the dominant whistler-mode waves, and therefore the particles which undergo 'significant diffusion' (see section 4.3 for a discussion on this topic, and our definition of 'significant diffusion'). The observed scattering in a preferred direction is an expected result due to the following argument. During a resonant interaction with a given parallel-propagating whistler-mode wave $\left(\omega_{k}<\left|\omega_{\text {ce }}\right|\right)$, then (in the rest frame of the wave) an electron will experience changes in the total kinetic energy, $\Delta E$, and the perpendicular kinetic energy, $\Delta E_{\perp}$, that are related according to

$$
\Delta E_{\perp} / \Delta E=\left|\omega_{\text {ce }}\right| / \omega_{k}>1,
$$

(e.g. see equation (36) in Brice [1964]). Equation (9) implies that (in the rest frame of the wave) for a given positive change in the electron energy $(\Delta E>0)$, such that

$$
\Delta E=\Delta E_{\perp}+\Delta E_{\|},
$$

then the perpendicular energy must increase by a greater amount, i.e. $\Delta E_{\perp}>\Delta E$. Therefore the parallel energy must decrease $\left(\Delta E_{\|}<0\right)$. This therefore implies that the pitch angle must increase. One can use an exactly analogous argument using equation (9) to conclude that a decrease in the energy of an electron $(\Delta E<0)$ is consistent with a decrease of the electron pitch angle. These, and other, helpful observations are summarized in Table 1. of Brice [1964], and can also be seen clearly in Figure 1. from Kennel and Petschek [1966].

Furthermore, we see from Figure 5 that the particle population spreads from an initial 'top-hat' sample, into a Gaussian-type distribution. This property seems broadly consistent with the diffusive paradigm, in which initially localized distributions spread into Gaussian distributions with ever greater widths (variances).

\subsection{The diffusive hypothesis}

The direct evaluation of the nature of diffusion in response to a given wave spectrum, relies on both the plasma and wave characteristics being quasi-static for the time considered, $\Delta t$ [Schulz and Lanzerotti, 1974]. Further to this requirement, we propose some additional constraints that are described by the following hierarchy of timescales,

$$
\tau_{\text {wave }} \ll \Delta t \leq \tau_{B(\omega, k)}, \tau_{n, B_{0}}, \tau_{\text {local }},
$$

that should be satisfied in order for one to directly measure properties of the diffusion for a given $(E, \alpha)$ bin. Equation (10) states that one can meaningfully evaluate diffusion due to interactions with a given wave frequency over timescales: (i) significantly larger than $\tau_{\text {wave }}=1 / f_{\text {wave }}$; (ii) smaller than those for which one observes variations in the wave spectrum $\left(\tau_{B(\omega, k)}\right)$; (iii) and smaller than those for which one observes variations in the the background magnetic field and number density $\left(\tau_{n, B_{0}}\right)$. These conditions hold for our experiment.

Furthermore, in order to be able to consider the rate of diffusion as a function of $E$ and $\alpha$ over some timescale $\Delta t$, each given sub-population (or bin) of particles must remain localized to the same given region of $(E, \alpha)$ space for the duration of $\Delta t$. To be clear, consider one particular bin composed of $l=1,2, \ldots, N$ particles, with initial values binned 

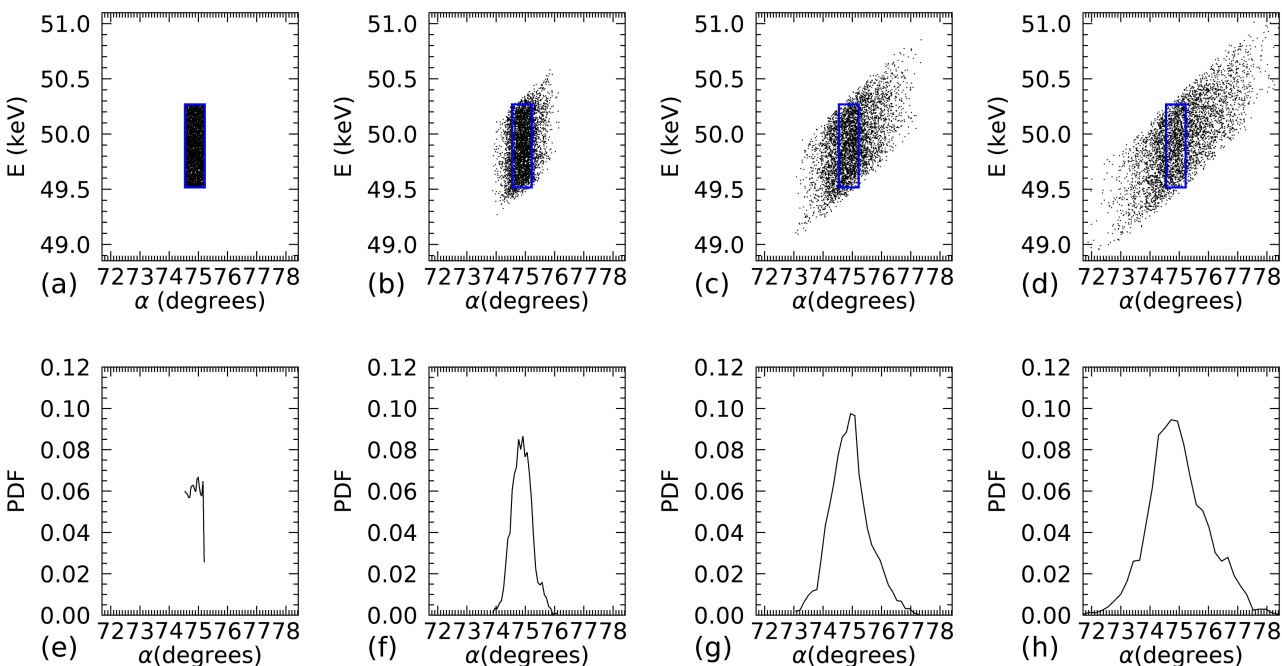

Figure 5. The diffusive response for 4444 electrons, within a bin roughly centred on $75^{\circ}$ and $50 \mathrm{keV}$. (a)-(d) plot electron $E, \alpha$ values at $\tilde{t}=0, T / 3,2 T / 3$ and $\tilde{t}=T$ respectively. (e)-(h) plot pitch angle distributions of the given particles at those times.

according to $\left[\left(E_{\mathrm{bin}, \min }<E_{l}(t=0)<E_{\mathrm{bin}, \max }\right),\left(\alpha_{\mathrm{bin}, \min }<\alpha_{l}(t=0)<\alpha_{\text {bin, } \max }\right)\right]$. Then, in order to be able to measure diffusion for that given bin, we require

$$
\begin{aligned}
E_{\text {bin, } \min } & <\left\langle E_{l}\right\rangle(\tilde{t})<E_{\text {bin, } \max }, \\
\alpha_{\text {bin, } \min } & <\left\langle\alpha_{l}\right\rangle(\tilde{t})<\alpha_{\text {bin, } \max } .
\end{aligned}
$$

We define the timescale over which $<\left\langle E_{l}\right\rangle(t)$ and $<\left\langle\alpha_{l}\right\rangle(\tilde{t})$ satisfy the above constraints in a given bin as $\tau_{\text {local }}$. Therefore, if satisfied, then the electrons in the given bin undergo negligible advection in $(E, \alpha)$ space over the timescale $\tau_{\text {local }}$.

Figure (6) plots a normalized measure of local advection in phase-space,

$$
d \alpha(E, \alpha):=\frac{\Delta\left\langle\alpha_{l}\right\rangle}{\left|\operatorname{bin}\left(\alpha_{l}\right)\right|} .
$$

Here, $\Delta\left\langle\alpha_{l}\right\rangle=\left\langle\alpha_{l}\right\rangle(\tilde{t}=T)-\left\langle\alpha_{l}\right\rangle(\tilde{t}=0)$, and $|\operatorname{bin}(\alpha)|=\alpha_{\text {bin, } \max }-\alpha_{\text {bin, } \min }$. The over-plotted white curves mark the values of energy and pitch angle that are (according to equations (4) and (5)) in " $n=-1$ resonance" with waves of frequency $f_{l c}$ ('dash'), $f_{u c}$ ('dash-dot'), and $\left(f_{l c}+f_{u c}\right) / 2$ ('solid'). We can see that the largest values of $d \alpha$ are localized to regions within the boundaries of the resonance curves, and have maximum size less than $0.5(\max (|d \alpha|) \approx 0.36)$. Therefore, for this choice of binning, all electron subpopulations remain localized to their given pitch-angle bins for the duration of the interaction that we consider, $0<\tilde{t}<T$. Therefore an evaluation of diffusive properties is valid, i.e. timescales are in agreement with equation (10) and the diffusive hypothesis holds over the given timescale $\Delta t=460 t_{c e}$. We have also constructed a similar plot for $d E$, which shows qualitatively similar results $(\max (|d E|) \approx 0.25)$, and so sub-populations also remain localized to their given energy bins.

\subsection{Nature of the diffusion}

As described at the beginning of Section 3, the calculation of a diffusion coefficient (e.g. $D_{\alpha \alpha}$ ) within the normal diffusive paradigm assumes that the variances of electron 


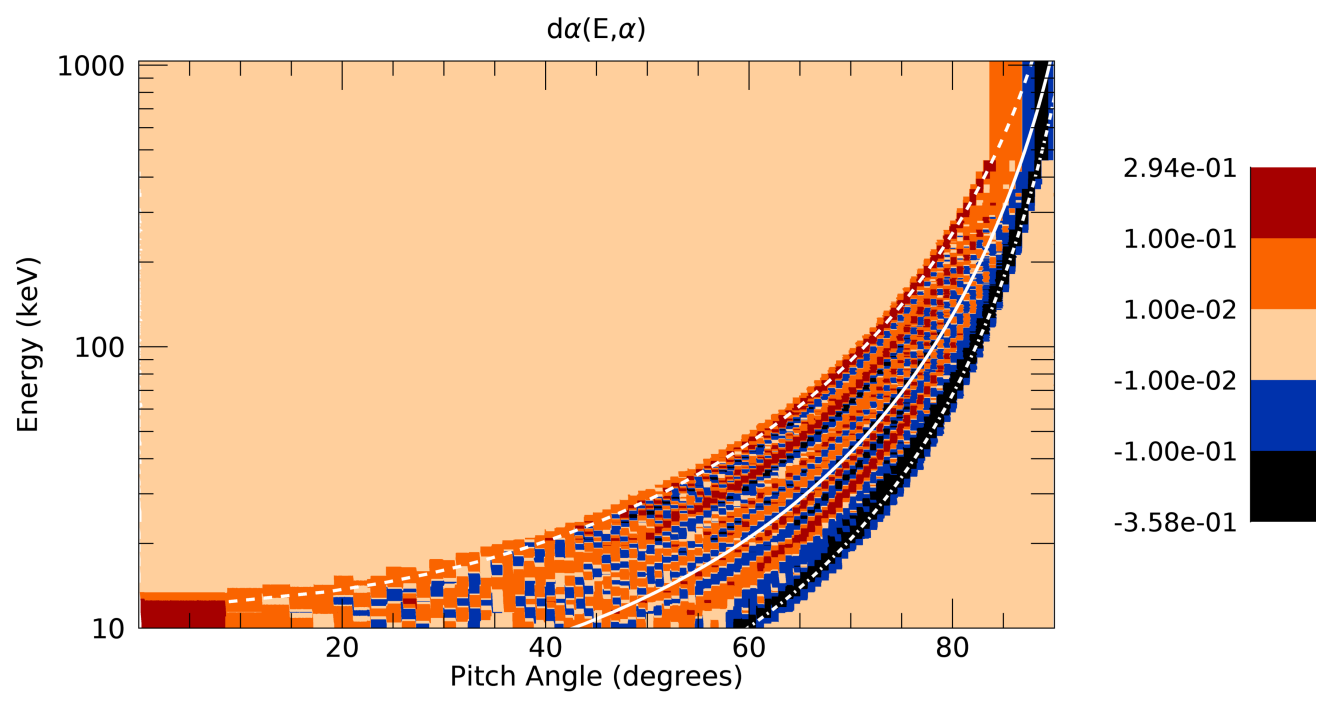

Figure 6. $d \alpha(E, \alpha)$, for each $(E, \alpha)$ bin in the experiment (see equation (12)). The over-plotted white curves mark the values of energy and pitch angle that are (according to equations (4) and (5)) in " $n=-1$ resonance" with waves of frequency $f_{l c}$ ('dash'), $f_{u c}$ ('dash-dot'), and $0.5\left(f_{l c}+f_{u c}\right)$ ('solid').

pitch-angle distributions within a given bin grow linearly with time. In this case, the diffusion coefficient is given by equation (8), and defines the slope of the line according to $\operatorname{var}\left(\alpha_{l}\right)=2 D_{\alpha \alpha} \Delta t$. Prior to calculating diffusion coefficients, it is therefore important to check whether the data supports this implicit assumption of 'variances that grow linearly in time'.

Figures 7(a)-(c) plot the evolution of $\operatorname{var}\left(\alpha_{l}\right)$ for three different example bins. These bins are given by $(E, \alpha)=\left(20 \mathrm{keV}, 45^{\circ}\right)\left(300 \mathrm{keV}, 82^{\circ}\right)$, and $\left(50 \mathrm{keV}, 75^{\circ}\right)$. The asterisks mark the directly extracted particle data, and the solid black lines mark curve fits. The curve-fitting method is described in Appendix B. For the purposes of presentation, the particle variance curves are translated so that $\operatorname{var}\left(\alpha_{l} ; \tilde{t}=0\right)=0$. An instantaneous measure of the rate of diffusion could be considered to be $d\left(\operatorname{var}\left(\alpha_{l}\right)\right) / d \tilde{t}$. Figure 7(b) shows an example for which this rate of diffusion appears to be roughly constant in time. However, figures 7(a) and 7(c) show examples for which the rate appears, respectively, to be slowing down and speeding up as time passes. This time-dependent rate of diffusion is an interesting feature, and does not (at first glance) appear consistent with the assumptions of normal diffusion theory. Diffusive theories that move beyond the assumption of normal diffusion (sometimes called 'anomalous diffusion') have many applications, and not only in space physics (e.g. see Bouchaud and Georges [1990]; Metzler and Klafter [2000]; Zaslavsky [2002]; Perrone et al. [2013]; Zimbardo et al. [2015]). Anomalous diffusion theory essentially allows for the variance of a given parameter $X$ to evolve according to a power-law

$$
\operatorname{var}\left(X_{l}\right) \propto t^{a},
$$

for $0<a<\infty$, and for which $a<1$ denotes 'sub-diffusion', $a \approx 1$ denotes 'normal diffusion', and $a>1$ denotes 'super-diffusion'. Using this interpretation, figures 7(a)-(c) present values $a=0.52, a=0.92$ and $a=2.21$ respectively (suggesting sub-, normal- and super-diffusion respectively). It is interesting to see that the diffusion observed does not always follow normal diffusive behaviour over the time-scales $0<\tilde{t}<T$. Each sub-figure also includes a blue solid line, obtained via imposing a linear fit to the raw data over the second half of the wave-particle interaction, $T / 2<\tilde{t}<T$. These straight lines are a good 
fit in each case. This suggests that normal diffusion is observed in all three cases, but only after an initial transient phase during which anomalous diffusion occurred.

Figure 8 plots the evolution of $\operatorname{var}(\alpha)$ for a bin roughly centred on $(E, \alpha)=\left(9.2 \mathrm{keV}, 40^{\circ}\right)$. This sub-population of particles is resonant with waves of frequency $f=0.3 f_{\text {ce }}$, and represents a direct comparison with the main example plotted by Tao et al. [2011] (figure 2 in that paper). The black and blue solid lines represent the same curve fits as applied to figure 7. The solid green line represents the theoretical evolution of $\operatorname{var}(\alpha)$, as inferred by using the value of $D_{\alpha \alpha}$ as predicted from the PADIE code [Glauert and Horne, 2005] using input plasma and wave parameters as detailed in this paper. As a reminder, note that $\operatorname{var}(\alpha)=2 \Delta t D_{\alpha \alpha}$ under the assumption of normal diffusion. The diffusion index for this bin is given by $a=1.15$ and therefore classified as normal diffusion. We see a very good agreement between the theoretical diffusion coefficient and our directly extracted data on the diffusive response for this particular energy and pitch-angle bin. We will follow up on direct comparisons between $D_{\alpha \alpha}$ as predicted from the PADIE code and diffusive response in our $\mathrm{PiC}$ experiments in a separate paper that will include all energy and pitch-angle bins.

Figure 9 presents contour plots of the diffusion index, $a$, for each $(E, \alpha)$ bin, as defined in equation (13). We remind that we calculate the value $a$ for each and every bin over all phase space $(0<\alpha<90,9 \mathrm{keV}<E<1 \mathrm{MeV})$. The rate of diffusion that is observed is a function of phase-space, and we observe very weak diffusion in some bins. We do not present the value of $a$ when diffusion is very weak, and instead mask those cases with black. For this purpose, a measure of the strength of the diffusion is calculated as follows. We calculate the total change in the variance of the pitch-angle distributions within each bin,

$$
D_{\alpha \alpha, \operatorname{proxy}}(E, \alpha)=\frac{\operatorname{var}\left(\alpha_{l} ; \tilde{t}=T\right)-\operatorname{var}\left(\alpha_{l} ; \tilde{t}=0\right)}{2 T} .
$$

Significant diffusion is deemed to have occurred within a given bin, if the value of this diffusion proxy satisfies

$$
D_{\alpha \alpha, \operatorname{proxy}}(E, \alpha)>10^{-2} \max _{E, \alpha}\left\{D_{\alpha \alpha, \operatorname{proxy}}(E, \alpha)\right\} .
$$

If significant diffusion does occur, then we describe its nature as 'sub-diffusion' if $<a<$ 0.67 by using yellow, as normal diffusion if $0.67<a<1.5$ by using pink, or as 'superdiffusion' if $a>1.5$ by using dark-red. These bounds of $a<a_{\text {sub }}=2 / 3$ and $a>a_{\text {super }}=$ $1 / a_{\text {sub }}$ were chosen in order to classify sub- and super-diffusion relatively strictly. Any regions for which the curve-fitting routine failed, are represented by missing data (white). As was done for figure 6, the over-plotted cyan curves mark the values of energy and pitch angle that are in " $n=-1$ resonance" with the driven waves. Furthermore, the locations of the bins represented in figure 7(a)-(c) are marked by cyan symbols: " $<$ ", " $\star$ "and ">" respectively. Figure 9(a) shows the value of $a$ that is calculated over the entire waveparticle interaction $(0<t<T)$. One clear observation is that the regions of significant diffusion, as defined by equation (15), are almost exclusively localized within the " $n=-1$ resonance" regions of phase space. Figure 9(a) also shows two well-defined region of suband super-diffusive behaviour. The sub-diffusive region is found at 'lower-frequency' resonances, and predominantly lower energies $(<100 \mathrm{keV})$; whereas the super-diffusive behavior is observed for 'higher-frequency' resonances, and across the entire energy range. Out of all bins in which significant diffusion was observed, 7.2\% show sub-diffusion, $45 \%$ show normal-diffusion, and $47.8 \%$ show super-diffusion.

Figure 9(b) shows the same quantity as figure 9(a), but calculated over the secondhalf of the interaction $(T / 2<\tilde{t}<T)$. This plot is motivated by the hypothesis made after analysis of figures 7(a)-(c), that an initial transient period of varying diffusion rates gives way to normal diffusion in the latter half of the experiment. We indeed see from figure 9(b) that a smaller proportion of the plot (i.e. fewer bins) displays the sub- and superdiffusive behaviour. This observation is consistent with the observations made of the data 
in figures 7(a)-(c): during $0<\tilde{t}<T / 2$ we observe an initial transient phase during which different kinds of diffusive responses are possible; later on (during $T / 2<\tilde{t}<T$ ) we observe a shift towards a normal-diffusive response. Out of all bins in which meaningful diffusion was observed, 2.9\% show sub-diffusion, $86.7 \%$ show normal-diffusion, and $10.4 \%$ show super-diffusion during the second half of the numerical experiment.

\section{Discussion}

We use the EPOCH particle-in-cell code [Arber et al., 2015] to track electron pitchangle diffusion due to interactions with whistler-mode waves. There are two main novel features to our approach: (i) we consider diffusion using a PiC code as a boundary value problem, i.e. we excite specific wave modes at the boundary, as opposed to considering an initial-value problem in which one typically considers waves that grow from an initially unstable distribution (e.g. see Katoh and Omura [2006]; Omura et al.; Hikishima et al. [2009]; Omura et al. [2009]; Omidi et al. [2010, 2011]; Katoh and Omura [2013]; Camporeale [2015]; Camporeale and Zimbardo [2015]; Silva et al. [2017]; Ratcliffe and Watt [2017]; Katoh et al. [2018]); (ii) by considering the response of a distribution of electrons, we track the diffusion in energy and pitch angle space across the entire phase-space domain, in contrast to some previous similar studies of diffusion (e.g. see Tao et al. [2011, 2012a]; Camporeale and Zimbardo [2015]) that considered resonant particles only. These novel features allow us to, respectively: (i) consider a 'quasi-static' system, in which the background plasma and whistler-mode wave spectra are roughly time-independent; (ii) derive characteristics of the diffusive response for all electrons, including those that are non-resonant with the waves and typically not expected to strongly interact.

In this first study, we model the background plasma as a $0.1 \mathrm{eV}$ isotropic cold population. We use this approach to benchmark our novel method with expected results, before studying more realistic 'radiation belt' background plasmas in the future, with 'warm' and 'hot' anisotropic electron populations (see e.g. Denton et al. [2010]). We also make the assumption of spatially 1D dynamics, thereby permitting only parallel and anti-parallel wave propagation, as well as a homogeneous background magnetic field. This considerable simplification is done in order to benchmark with previous work [Tao et al., 2011], and with a mind to a systematic future program of work with more realistic magnetic field geometries, wave-normal-angle spectra, and/or more 'exotic' wave modes and amplitudes. In order to properly understand each effect, it is necessary to first understand the experimental response in the most simple of circumstances, and then implement additions in isolation. We have compared our results for pitch angle diffusion with the example presented by Tao et al. [2011], using results from the PADIE code, and we see very good agreement. A more comprehensive comparison of the diffusive response with the predictions of QLT is beyond the scope of this paper, but will be addressed explicitly in future papers in this series.

Extremely low levels of background noise have been enabled by using the $\delta F$ mode in EPOCH. This, in addition to the linear envelope applied to the wave driver, allows for a highly effective wave-driving mechanism for whistler-mode waves, with an excellent signal-noise-ratio. We have verified that the wave power is well localized to follow the cold plasma dispersion relation. A large number of tracer particles were embedded within the $\mathrm{PiC}$ domain $\left(\approx 10^{8}\right.$ electrons) by using the EPOCH tracer functionality. This provides good statistics with which we characterize the electron diffusion over the entire energy and pitch angle domain. The dominant diffusion is clearly seen to correspond to those particles that are in ' $n=-1$ ' resonance with the driven wave mode, as expected by QLT. Diffusive effects outside the resonant regions of phase-space are at most $1 \%$ as significant as the dominant resonant effects. Therefore, for this experiment, non-resonant interactions are of little importance (unlike in e.g. Camporeale [2015]). This feature is an explicit requirement of QLT, and seems sensible given the simplified nature of the experimental setup. 
Future investigations will seek to determine those circumstances under which nonlinear effects become more important.

One interesting feature of the diffusion is observed for early times; namely, a timedependent rate of diffusion. The variances of pitch-angle distributions of given electron sub-populations do not always grow linearly with time during the first half of the experiment (see figures 7 and 9). We discuss this result in the context of 'anomalous' diffusion theories (e.g. see Bouchaud and Georges [1990]; Metzler and Klafter [2000]; Zaslavsky [2002]; del Castillo-Negrete et al. [2004]; Perrone et al. [2013]; Zimbardo et al. [2015]), that are known to play a role in various space and astrophysical plasma contexts. However, at later times, normal diffusion (Brownian motion) is seen to dominate, during which time the variances grow with a linear rate. This latter result is consistent with the implicit assumptions of QLT, and suggests that in order to construct meaningful diffusion coefficients from such PiC experiments, one may have to consider how to appropriately treat this initial transient phase (see examples of possible methods to treat time-dependent diffusion rates in e.g. Degeling et al. [2007]).

\section{Summary}

In this first paper of a series, we analyze the nature of the electron pitch-angle diffusion due to interactions with broadband and incoherent whistler-mode waves. The most significant technical development is a proof-of-concept for the novel method used. Namely, an analysis of the nature of electron diffusion over all phase-space due to specified whistlerwave modes, using a particle-in-cell method to construct a boundary-value problem. This analysis is enabled by direct extraction of particle data from numerical experiments performed using the EPOCH code. The numerical experiment was intended to both resemble and build upon the test-particle experiments performed by Tao et al. [2011]. As such, we drove an incoherent spectrum of whistler-mode waves into a simple cold plasma with uniform background magnetic field, and tracked the diffusive response of $\approx 10^{8}$ electrons for $460 \mathrm{t}_{\mathrm{ce}}$, as a function of energy and pitch angle. We make the following observations of our experiments:

1. The strength of the diffusive response is found to be a function of energy and pitch angle, as is expected using quasilinear diffusion theory. The diffusion is strongest in regions of energy-pitch angle space that are in the $n=-1$ resonance with the dominant wave signal. This is not a new result, but a required one, since the only wave-particle resonance that is possible in our experiment is the $n=-1$ case. However, it is an important benchmarking criteria that our novel method must satisfy, and therefore it is important to check and present.

2. Non-resonant interactions are observed to be of little consequence in this case. This feature is directly observed in our numerical experiments, and is not a priori assumed. It will be interesting to check how this feature changes in future experiments.

3. When considered over the entire duration of the wave particle interaction for particular regions of energy-pitch angle space, the nature of the diffusive response is observed in some regions of phase-space to be: (a) 'normal', i.e. it is Einsteinian/Brownian (for which the variances of energy or pitch angle grow linearly with time, as is implicit in QLT); (b) or 'anomalous', i.e. it is either super- or sub-diffusive (for which the variances of energy or pitch angle grow at either a faster or slower relative rate, respectively)

4. When considered over the second half of the wave-particle interaction, we observe that a larger proportion of phase-space exhibits a normal diffusive response due to the wave-particle interaction 
5. A preliminary analysis of the strength of pitch-angle diffusion for a given region of energy and pitch angle space demonstrates consistency with the results presented by Tao et al. [2011], and the results of the PADIE code.

The results presented in this paper effectively benchmark our techniques against other treatments that consider the response of test-particles to whistler-mode waves (e.g. see Tao et al. [2011]). This work motivates the following future investigations on: (i) the strength and nature of diffusion as a function of driving wave amplitude; (ii) the difference in the plasma response when more realistic radiation belt plasmas are modelled, i.e. those with the fractional warm components that can be found in Earth's inner magnetosphere. It will also be interesting to consider methods that allow for a analysis of a fully self-consistent electron response to whistler-mode waves, i.e. to move beyond the mixed 'PiC - test-particle approach' that we employed in this paper, and therefore beyond any of its inherent limitations.

Beyond that, there are many questions remaining that motivate other future studies. Namely, how do the results presented in this paper change when modelling other manifestations of whistler-mode waves (e.g. monochromatic waves), other wave spectra (e.g. with a much wider wave-normal angle spectrum). It will also be important to study the diffusive response in spatially two-dimensional plasmas, with both homogeneous and inhomogeneous background magnetic fields. Oblique waves can only propagate in a numerical experiment that has more than one spatial dimension, and are known to be relevant for some properties of whistler-mode wave dynamics in the radiation belts (e.g. see Artemyev et al. [2016a]; Ratcliffe and Watt [2017]). All of these effects should be expected to produce qualitatively different diffusive responses.

\section{A: The EPOCH particle-in-cell code}

We use version 4.16 of the Extendable PiC Open Collaboration (EPOCH) code in one spatial dimension ('EPOCH1D'). EPOCH is an explicit [Birdsall and Langdon, 2004], relativistic and charge-conserving PiC code [Arber et al., 2015], using Villasenor and Buneman current deposition [Villasenor and Buneman, 1992]. EPOCH solves Maxwell's equations combined with the equations of motion for charged particles in an EM field to provide a direct simulation of collisionless plasma. Since EPOCH uses an explicit scheme, numerical stability requirements dictate that timesteps, $\Delta t$, are limited by the usual CFL condition, and the resolution of electron plasma waves, for a given grid discretization $\Delta x$. EPOCH1D therefore sets the time scale as the most restrictive of constraints set by: the CFL condition $\left(\Delta t_{\mathrm{CFL}}=\Delta x / c\right)$; the inverse plasma frequency, (at the beginning of the simulation); and the inverse 'laser' frequency (the term 'laser' refers to an electromagnetic wave driver), according to

$$
\Delta t<\min \left(\Delta t_{\mathrm{CFL}}, 1 /\left(2 f_{\mathrm{BG}}\right), 1 /\left(2 f_{\text {laser }}\right)\right),
$$

for $f_{\mathrm{BG}}=\left|\omega_{\mathrm{BG}} /(2 \pi)\right|$ the ordinary frequency according to the Bohm-Gross dispersion relation [Bohm and Gross, 1949], and $f_{\text {laser }}$ the minimum laser frequency. All quantities in EPOCH are given in normal SI units.

EPOCH allows users to run in ' $\delta F$ mode'. In general terms, if we consider a plasma population as being described by a distribution function of the form $F=F_{0}(\mathbf{x}, \mathbf{v})+\delta F(\mathbf{x}, \mathbf{v}, t)$ (with $F_{0}$ either an isotropic or anisotropic Maxwellian distribution function), then the $\delta F$ method (e.g. see Sydora [1999]) can achieve a reduction in PiC noise of the order of $\sim|\delta F| / F$, for all other settings left unchanged. Hence, this method is particularly useful if $F$ is close to an (an-)isotropic Maxwellian distribution function.

Instructions on how to download and run EPOCH are given in supplementary information (S1). 


\section{B: Curve-fitting procedure}

Here we describe the curve-fitting procedure, used to determine the diffusion index $a$, as shown in figures 7, 8 and 9. In each $(E, \alpha)$ bin, a time-series of the quantity

$$
V_{\alpha}(\tilde{t})=\frac{\operatorname{var}\left(\alpha_{l} ; \tilde{t}\right)-\operatorname{var}\left(\alpha_{l} ; \tilde{t}=0\right)}{\operatorname{var}\left(\alpha_{l} ; \tilde{t}=0\right)},
$$

is calculated. We track $V_{\alpha}$ instead of just the variance, so that all time-series are ordinated according to similar scales, and are initialized at zero. This also helps with the curvefitting procedure which is performed as follows:

(i) First, we test to see whether or not any 'significant diffusion' occurs in each bin, using the rule defined by equation (15). (ii) If significant diffusion occurred then curvefitting of the time-series, $V_{\alpha}$, is implemented, to the test function,

$$
y=c_{0}+c_{1} x^{c_{2}} .
$$

For a given initial estimate of the vector $\mathbf{c}=\left[c_{0}, c_{1}, c_{2}\right]$, the standard curve-fitting routine uses a gradient-expansion least-squares method, and returns a successful result when the relative decrease in chi-squared is less than $10^{-3}$ in a given iteration. The routine returns a fail if convergence is not reached after a large number of iterations, or if the chisquared value increases without bounds. Note that we classify 'successful' outputs of the curve-fitting algorithm as 'failures' if $c_{2}>10$, in order to neglect spuriously high values. Finally, curve-fitting could only be considered a success if $\left|y(x=0) / y\left(x=x_{\max }\right)\right|<0.5$.

(iii) For each bin, we employ this curve fitting routine for 100 different initial guesses of $\mathbf{c}$, and record the output values of all 'successful' attempts. We then select the output vector that minimizes the mean deviation between the curve fit and the data. In theory, normal diffusion would give $c_{2} \approx 1$, with the coefficient $c_{1}$ directly proportional to $D_{\alpha \alpha}$, and $c_{0}=0$.

\section{Acknowledgments}

The supporting information provides: (S1) basic instructions on how to run the same experiment that is presented in the main article; (S2) the contents of the input text file, used for the numerical experiment that is presented in the main article. This information will enable readers to locally generate the same experimental data as was considered in the main article.

This research was supported by the Natural Environment Research Council (NERC) Highlight Topic Grant \#NE/P017274/1 (Rad-Sat).This work was in part funded by the UK EPSRC grants EP/G054950/1, EP/G056803/1, EP/G055165/1 and EP/ M022463/1.This work was in part performed using the Cambridge Service for Data Driven Discovery (CSD3), part of which is operated by the University of Cambridge Research Computing on behalf of the STFC DiRAC HPC Facility (www.dirac.ac.uk). The DiRAC component of CSD3 was funded by BEIS capital funding via STFC capital grants ST/P002307/1 and ST/R002452/1 and STFC operations grant ST/R00689X/1. DiRAC is part of the National e-Infrastructure.This work was in part performed using the Research Processing Service (Met-Cluster) and the Reading Academic Computing Cluster (RACC) at the University of Reading.

The authors acknowledge helpful conversations with M. Balikhin, A.W. Degeling, F. Effenberger, C. Forsyth, R.B. Horne, T. Neukirch, I.J. Rae and S. Troscheit.

\section{References}

Albert, J. M. (2001), Comparison of pitch angle diffusion by turbulent and monochromatic whistler waves, Journal of Geophysical Research: Space Physics, 106(A5), 8477-8482, doi:10.1029/2000JA000304. 
Albert, J. M. (2002), Nonlinear interaction of outer zone electrons with vlf waves, Geophysical Research Letters, 29(8), 116-1-116-3, doi:10.1029/2001GL013941.

Albert, J. M. (2010), Diffusion by one wave and by many waves, Journal of Geophysical Research: Space Physics, 115(A3), doi:10.1029/2009JA014732.

Arber, T. D., K. Bennett, C. S. Brady, A. Lawrence-Douglas, M. G. Ramsay, N. J. Sircombe, P. Gillies, R. G. Evans, H. Schmitz, A. R. Bell, and C. P. Ridgers (2015), Contemporary particle-in-cell approach to laser-plasma modelling, Plasma Physics and Controlled Fusion, 57(11), 1-26.

Artemyev, A., O. Agapitov, D. Mourenas, V. Krasnoselskikh, V. Shastun, and F. Mozer (2016a), Oblique whistler-mode waves in the earth's inner magnetosphere: Energy distribution, origins, and role in radiation belt dynamics, Space Science Reviews, 200(1), 261-355, doi:10.1007/s11214-016-0252-5.

Artemyev, A. V., A. I. Neishtadt, A. A. Vasiliev, and D. Mourenas (2016b), Kinetic equation for nonlinear resonant wave-particle interaction, Physics of Plasmas, 23(9), 090,701, doi:10.1063/1.4962526.

Artemyev, A. V., A. I. Neishtadt, A. A. Vasiliev, and D. Mourenas (2017), Probabilistic approach to nonlinear wave-particle resonant interaction, Phys. Rev. E, 95, 023,204, doi: 10.1103/PhysRevE.95.023204.

Artemyev, A. V., A. I. Neishtadt, A. A. Vasiliev, and D. Mourenas (2018), Longterm evolution of electron distribution function due to nonlinear resonant interaction with whistler mode waves, Journal of Plasma Physics, 84(2), 905840,206, doi: $10.1017 / \mathrm{S} 0022377818000260$.

Birdsall, C., and A. Langdon (2004), Plasma Physics via Computer Simulation, Series in Plasma Physics and Fluid Dynamics, Taylor \& Francis.

Bohm, D., and E. P. Gross (1949), Theory of plasma oscillations. a. origin of medium-like behavior, Phys. Rev., 75, 1851-1864, doi:10.1103/PhysRev.75.1851.

Bortnik, J., R. M. Thorne, and N. P. Meredith (2008a), The unexpected origin of plasmaspheric hiss from discrete chorus emissions, Nature, 452(7183), 62-66, doi: 10.1038/nature06741.

Bortnik, J., R. M. Thorne, and U. S. Inan (2008b), Nonlinear interaction of energetic electrons with large amplitude chorus, Geophysical Research Letters, 35(21), doi: 10.1029/2008GL035500.

Bouchaud, J.-P., and A. Georges (1990), Anomalous diffusion in disordered media: Statistical mechanisms, models and physical applications, Physics Reports, 195(4), 127 - 293, doi:https://doi.org/10.1016/0370-1573(90)90099-N.

Breneman, A., C. Cattell, J. Wygant, K. Kersten, L. B. Wilson, S. Schreiner, P. J. Kellogg, and K. Goetz (2011), Large-amplitude transmitter-associated and lightning-associated whistler waves in the earth's inner plasmasphere at $1<2$, Journal of Geophysical Research: Space Physics, 116(A6), doi:10.1029/2010JA016288.

Brice, N. (1964), Fundamentals of very low frequency emission generation mechanisms, Journal of Geophysical Research (1896-1977), 69(21), 4515-4522, doi: 10.1029/JZ069i021p04515.

Camporeale, E. (2015), Resonant and nonresonant whistlers-particle interaction in the radiation belts, Geophysical Research Letters, 42(9), 3114-3121, doi: 10.1002/2015GL063874.

Camporeale, E., and G. Zimbardo (2015), Wave-particle interactions with parallel whistler waves: Nonlinear and time-dependent effects revealed by particle-in-cell simulations, Physics of Plasmas, 22(9), 092,104, doi:10.1063/1.4929853.

Cattell, C., J. R. Wygant, K. Goetz, K. Kersten, P. J. Kellogg, T. von Rosenvinge, S. D. Bale, I. Roth, M. Temerin, M. K. Hudson, R. A. Mewaldt, M. Wiedenbeck, M. Maksimovic, R. Ergun, M. Acuna, and C. T. Russell (2008), Discovery of very large amplitude whistler-mode waves in earth's radiation belts, Geophysical Research Letters, 35(1), doi:10.1029/2007GL032009. 
Chen, H., X. Gao, Q. Lu, Y. Ke, and S. Wang (2017), Lower band cascade of whistler waves excited by anisotropic hot electrons: One-dimensional pic simulations, Journal of Geophysical Research: Space Physics, 122(10), 10,448-10,457, doi: 10.1002/2017JA024513.

Chen, H., X. Gao, Q. Lu, J. Sun, and S. Wang (2018), Nonlinear evolution of counterpropagating whistler mode waves excited by anisotropic electrons within the equatorial source region: 1-d pic simulations, Journal of Geophysical Research: Space Physics, 123(2), 1200-1207, doi:10.1002/2017JA024850.

Chen, L., W. Li, J. Bortnik, and R. M. Thorne (2012), Amplification of whistlermode hiss inside the plasmasphere, Geophysical Research Letters, 39(8), doi: 10.1029/2012GL051488.

Chen, L., R. M. Thorne, J. Bortnik, W. Li, R. B. Horne, G. D. Reeves, C. A. Kletzing, W. S. Kurth, G. B. Hospodarsky, H. E. Spence, J. B. Blake, and J. F. Fennell (2014), Generation of unusually low frequency plasmaspheric hiss, Geophysical Research Letters, 4l(16), 5702-5709, doi:10.1002/2014GL060628.

Cully, C. M., J. W. Bonnell, and R. E. Ergun (2008), Themis observations of long-lived regions of large-amplitude whistler waves in the inner magnetosphere, Geophysical Research Letters, 35(17), doi:10.1029/2008GL033643.

Degeling, A., R. Rankin, K. Kabin, R. Marchand, and I. Mann (2007), The effect of ulf compressional modes and field line resonances on relativistic electron dynamics, Planetary and Space Science, 55(6), 731 - 742, doi:https://doi.org/10.1016/j.pss.2006.04.039, ultra-Low Frequency Waves in the Magnetosphere.

del Castillo-Negrete, D., B. A. Carreras, and V. E. Lynch (2004), Fractional diffusion in plasma turbulence, Physics of Plasmas, 11(8), 3854-3864, doi:10.1063/1.1767097.

Denton, M. H., J. E. Borovsky, and T. E. Cayton (2010), A density-temperature description of the outer electron radiation belt during geomagnetic storms, Journal of Geophysical Research: Space Physics, 115(A1), doi:10.1029/2009JA014183.

Fälthammar, C.-G. (1965), Effects of time-dependent electric fields on geomagnetically trapped radiation, Journal of Geophysical Research, 70(11), 2503-2516, doi: 10.1029/JZ070i011p02503.

Gao, X., Q. Lu, J. Bortnik, W. Li, L. Chen, and S. Wang (2016), Generation of multiband chorus by lower band cascade in the earth's magnetosphere, Geophysical Research Letters, 43(6), 2343-2350, doi:10.1002/2016GL068313.

Gao, X., Y. Ke, Q. Lu, L. Chen, and S. Wang (2017), Generation of multiband chorus in the earth's magnetosphere: 1-d pic simulation, Geophysical Research Letters, 44(2), 618-624, doi:10.1002/2016GL072251.

Glauert, S. A., and R. B. Horne (2005), Calculation of pitch angle and energy diffusion coefficients with the PADIE code, Journal of Geophysical Research, 110(A4), A04,206, doi:10.1029/2004JA010851.

Glauert, S. A., R. B. Horne, and N. P. Meredith (2014), Three-dimensional electron radiation belt simulations using the bas radiation belt model with new diffusion models for chorus, plasmaspheric hiss, and lightning-generated whistlers, Journal of Geophysical Research: Space Physics, 119(1), 268-289, doi:10.1002/2013JA019281.

Hikishima, M., S. Yagitani, Y. Omura, and I. Nagano (2009), Full particle simulation of whistler-mode rising chorus emissions in the magnetosphere, JGR: Space Physics.

Horne, R. B., R. M. Thorne, Y. Y. Shprits, N. P. Meredith, S. A. Glauert, A. J. Smith, S. G. Kanekal, D. N. Baker, M. J. Engebretson, J. L. Posch, M. Spasojevic, U. S. Inan, J. S. Pickett, and P. M. E. Decreau (2005a), Wave acceleration of electrons in the Van Allen radiation belts, Nature, 437, 227-230, doi:10.1038/nature03939.

Horne, R. B., R. M. Thorne, S. A. Glauert, J. M. Albert, N. P. Meredith, and R. R. Anderson (2005b), Timescale for radiation belt electron acceleration by whistler mode chorus waves, JGR: Space Physics, 110, A03225.

Hudson, M. K., S. R. Elkington, J. G. Lyon, and C. C. Goodrich (2000), Increase in relativistic electron flux in the inner magnetosphere: ULF wave mode structure, Advances 
in Space Research, 25(12), 2327-2337, doi:10.1016/S0273-1177(99)00518-9.

Katoh, Y., and Y. Omura (2006), A study of generation mechanism of VLF triggered emission by self-consistent particle code, JGR: Space Physics, 111, A12207.

Katoh, Y., and Y. Omura (2013), Effect of the background magnetic field inhomogeneity on generation processes of whistler-mode chorus and broadband hiss-like emissions, JGR: Space Physics), 118, 4189-4198.

Katoh, Y., Y. Omura, Y. Miyake, H. Usui, and H. Nakashima (2018), Dependence of generation of whistler mode chorus emissions on the temperature anisotropy and density of energetic electrons in the earth's inner magnetosphere, Journal of Geophysical Research: Space Physics, 123(2), 1165-1177, doi:10.1002/2017JA024801.

Kellogg, P. J., C. A. Cattell, K. Goetz, S. J. Monson, and L. B. Wilson (2011), Large amplitude whistlers in the magnetosphere observed with wind-waves, Journal of Geophysical Research: Space Physics, 116(A9), doi:10.1029/2010JA015919.

Kennel, C. F., and F. Engelmann (1966), Velocity space diffusion from weak plasma turbulence in a magnetic field, The Physics of Fluids, 9(12), 2377-2388, doi: $10.1063 / 1.1761629$.

Kennel, C. F., and H. E. Petschek (1966), Limit on stably trapped particle fluxes, Journal of Geophysical Research (1896-1977), 71(1), 1-28, doi:10.1029/JZ071i001p00001.

Langdon, A. (1970), Effects of the spatial grid in simulation plasmas, Journal of Computational Physics, 6(2), 247 - 267, doi:https://doi.org/10.1016/0021-9991(70)90024-0.

Lerche, I. (1968), Quasilinear theory of resonant diffusion in a magnetoactive, relativistic plasma, The Physics of Fluids, 11(8), 1720-1727, doi:10.1063/1.1692186.

Lyons, L. R. (1974), General relations for resonant particle diffusion in pitch angle and energy, Journal of Plasma Physics, 12(1), 45âĂŞ49, doi:10.1017/S0022377800024910.

Meredith, N. P., R. B. Horne, R. M. Thorne, and R. R. Anderson (2009), Survey of upper band chorus and ech waves: Implications for the diffuse aurora, Journal of Geophysical Research: Space Physics, 114(A7), doi:10.1029/2009JA014230.

Meredith, N. P., R. B. Horne, T. Kersten, W. Li, J. Bortnik, A. Sicard, and K. H. Yearby (2018), Global model of plasmaspheric hiss from multiple satellite observations, Journal of Geophysical Research: Space Physics, 123(6), 4526-4541, doi: 10.1029/2018JA025226.

Meredith, N. P., R. B. Horne, M. A. Clilverd, and J. P. J. Ross (2019), An investigation of vlf transmitter wave power in the inner radiation belt and slot region, Journal of Geophysical Research: Space Physics, O(ja), doi:10.1029/2019JA026715.

Metzler, R., and J. Klafter (2000), The random walk's guide to anomalous diffusion: a fractional dynamics approach, Physics Reports, 339(1), 1 - 77, doi: https://doi.org/10.1016/S0370-1573(00)00070-3.

Mourenas, D., X.-J. Zhang, A. V. Artemyev, V. Angelopoulos, R. M. Thorne, J. Bortnik, A. I. Neishtadt, and A. A. Vasiliev (2018), Electron nonlinear resonant interaction with short and intense parallel chorus wave packets, Journal of Geophysical Research: Space Physics, 123(6), 4979-4999, doi:10.1029/2018JA025417.

Northrop, T. G. (1963), Adiabatic Charged-Particle Motion, Reviews of Geophysics and Space Physics, 1, 283-304, doi:10.1029/RG001i003p00283.

Němec, F., O. SantolÃnk, M. Parrot, and C. J. Rodger (2010), Relationship between median intensities of electromagnetic emissions in the vlf range and lightning activity, Journal of Geophysical Research: Space Physics, 115(A8), doi:10.1029/2010JA015296.

Omidi, N., R. M. Thorne, and J. Bortnik (2010), Nonlinear evolution of emic waves in a uniform magnetic field: 1. hybrid simulations, Journal of Geophysical Research: Space Physics, 115(A12), doi:10.1029/2010JA015607.

Omidi, N., R. Thorne, and J. Bortnik (2011), Hybrid simulations of emic waves in a dipolar magnetic field, Journal of Geophysical Research: Space Physics, 116(A9), doi: 10.1029/2011JA016511.

Omura, Y., Y. Katoh, and D. Summers (), Theory and simulation of the generation of whistler-mode chorus, Journal of Geophysical Research: Space Physics, 113(A4), doi: 
10.1029/2007JA012622.

Omura, Y., N. Furuya, and D. Summers (2007), Relativistic turning acceleration of resonant electrons by coherent whistler mode waves in a dipole magnetic field, Journal of Geophysical Research: Space Physics, 112(A6), doi:10.1029/2006JA012243.

Omura, Y., M. Hikishima, Y. Katoh, D. Summers, and S. Yagitani (2009), Nonlinear mechanisms of lower-band and upper-band VLF chorus emissions in the magnetosphere, JGR: Space Physics.

Perrone, D., R. O. Dendy, I. Furno, R. Sanchez, G. Zimbardo, A. Bovet, A. Fasoli, K. Gustafson, S. Perri, P. Ricci, and F. Valentini (2013), Nonclassical transport and particle-field coupling: from laboratory plasmas to the solar wind, Space Science Reviews, 178(2), 233-270, doi:10.1007/s11214-013-9966-9.

Ratcliffe, H., and C. E. J. Watt (2017), Self-consistent formation of a 0.5 cyclotron frequency gap in magnetospheric whistler mode waves, Journal of Geophysical Research: Space Physics, 122(8), 8166-8180, doi:10.1002/2017JA024399, $2017 J A 024399$.

Reeves, G. D., H. E. Spence, M. G. Henderson, S. K. Morley, R. H. W. Friedel, H. O. Funsten, D. N. Baker, S. G. Kanekal, J. B. Blake, J. F. Fennell, S. G. Claudepierre, R. M. Thorne, D. L. Turner, C. A. Kletzing, W. S. Kurth, B. A. Larsen, and J. T. Niehof (2013), Electron Acceleration in the Heart of the Van Allen Radiation Belts, Science, 341(6149), 991-994.

Roederer, J., and H. Zhang (2013), Dynamics of Magnetically Trapped Particles: Foundations of the Physics of Radiation Belts and Space Plasmas, Astrophysics and Space Science Library, Springer Berlin Heidelberg.

Roederer, J. G., and S. Lejosne (2018), Coordinates for representing radiation belt particle flux, Journal of Geophysical Research: Space Physics, 123(2), 1381-1387, doi: 10.1002/2017JA025053.

Schulz, M., and L. Lanzerotti (1974), Particle diffusion in the radiation belts, Physics and chemistry in space, Springer-Verlag.

Silva, C. L., S. Wu, R. E. Denton, M. K. Hudson, and R. M. Millan (2017), Hybrid fluidparticle simulation of whistler-mode waves in a compressed dipole magnetic field: Implications for dayside high-latitude chorus, Journal of Geophysical Research: Space Physics, 122(1), 432-448, doi:10.1002/2016JA023446.

Silva, C. L., R. E. Denton, M. K. Hudson, R. M. Millan, K. Liu, and J. Bortnik (2018), Test-particle simulations of linear and nonlinear interactions between a 2-d whistlermode wave packet and radiation belt electrons, Geophysical Research Letters, 45(11), 5234-5245, doi:10.1029/2018GL077877.

Stix, T. (1992), Waves in Plasmas, American Inst. of Physics.

Summers, D. (2005), Quasi-linear diffusion coefficients for field-aligned electromagnetic waves with applications to the magnetosphere, Journal of Geophysical Research: Space Physics, 110(A8), doi:10.1029/2005JA011159.

Sydora, R. (1999), Low-noise electromagnetic and relativistic particle-in-cell plasma simulation models, Journal of Computational and Applied Mathematics, 109(1), 243 - 259, doi:https://doi.org/10.1016/S0377-0427(99)00161-2.

Tao, X., and J. Bortnik (2010), Nonlinear interactions between relativistic radiation belt electrons and oblique whistler mode waves, Nonlinear Processes in Geophysics, 17(5), 599-604, doi:10.5194/npg-17-599-2010.

Tao, X., J. Bortnik, J. M. Albert, K. Liu, and R. M. Thorne (2011), Comparison of quasilinear diffusion coefficients for parallel propagating whistler mode waves with test particle simulations, Geophysical Research Letters, 38(6), doi:10.1029/2011GL046787.

Tao, X., J. Bortnik, R. M. Thorne, J. M. Albert, and W. Li (2012a), Effects of amplitude modulation on nonlinear interactions between electrons and chorus waves, Geophysical Research Letters, 39(6), n/a-n/a, doi:10.1029/2012GL051202.

Tao, X., J. Bortnik, R. M. Thorne, J. M. Albert, and W. Li (2012b), Effects of amplitude modulation on nonlinear interactions between electrons and chorus waves, Geophysical Research Letters, 39(6), doi:10.1029/2012GL051202. 
Tao, X., J. Bortnik, J. Albert, R. Thorne, and W. Li (2013), The importance of amplitude modulation in nonlinear interactions between electrons and large amplitude whistler waves, Journal of Atmospheric and Solar-Terrestrial Physics, 99, 67 - 72, doi: https://doi.org/10.1016/j.jastp.2012.05.012, dynamics of the Complex Geospace System.

Thorne, R. M. (2010), Radiation belt dynamics: The importance of wave-particle interactions, Geophysical Research Letters, 37(22), doi:10.1029/2010GL044990.

Thorne, R. M., B. Ni, X. Tao, R. B. Horne, and N. P. Meredith (2010), Scattering by chorus waves as the dominant cause of diffuse auroral precipitation, Nature, 467, 943-946, doi:10.1038/nature09467.

Thorne, R. M., W. Li, B. Ni, Q. Ma, J. Bortnik, L. Chen, D. N. Baker, H. E. Spence, G. D. Reeves, M. G. Henderson, C. A. Kletzing, W. S. Kurth, G. B. Hospodarsky, J. B. Blake, J. F. Fennell, S. G. Claudepierre, and S. G. Kanekal (2013), Rapid local acceleration of relativistic radiation-belt electrons by magnetospheric chorus, Nature, 504, 411-414, doi:10.1038/nature12889.

Treumann, R. A., and W. Baumjohann (2001), Advanced Space Plasma Physics, Imperial College Press.

Tsiklauri, D. (2016), The effect of a longitudinal density gradient on electron plasma wake field acceleration, Proceedings of the Royal Society A: Mathematical, Physical and Engineering Sciences, 472(2196), 20160,630, doi:10.1098/rspa.2016.0630.

Tsurutani, B. T., and E. J. Smith (1977), Two types of magnetospheric elf chorus and their substorm dependences, Journal of Geophysical Research (1896-1977), 82(32), 5112_ 5128, doi:10.1029/JA082i032p05112.

Vainchtein, D., X.-J. Zhang, A. V. Artemyev, D. Mourenas, V. Angelopoulos, and R. M. Thorne (2018), Evolution of electron distribution driven by nonlinear resonances with intense field-aligned chorus waves, Journal of Geophysical Research: Space Physics, O(0), doi:10.1029/2018JA025654.

Villasenor, J., and O. Buneman (1992), Rigorous charge conservation for local electromagnetic field solvers, Computer Physics Communications, 69(2), 306 - 316, doi: https://doi.org/10.1016/0010-4655(92)90169-Y.

Wilson III, L. B., C. A. Cattell, P. J. Kellogg, J. R. Wygant, K. Goetz, A. Breneman, and K. Kersten (2011), The properties of large amplitude whistler mode waves in the magnetosphere: Propagation and relationship with geomagnetic activity, Geophys. Res. Lett, 38, doi:10.1029/2011GL048671.

Zaslavsky, G. (2002), Chaos, fractional kinetics, and anomalous transport, Physics Reports, 371(6), 461 - 580, doi:https://doi.org/10.1016/S0370-1573(02)00331-9.

Zhang, X.-J., R. Thorne, A. Artemyev, D. Mourenas, V. Angelopoulos, J. Bortnik, C. A. Kletzing, W. S. Kurth, and G. B. Hospodarsky (2018a), Properties of intense fieldaligned lower-band chorus waves: Implications for nonlinear wave-particle interactions, Journal of Geophysical Research: Space Physics, 123(7), 5379-5393, doi: 10.1029/2018JA025390.

Zhang, Z., L. Chen, X. Li, Z. Xia, R. A. Heelis, and R. B. Horne (2018b), Observed propagation route of vlf transmitter signals in the magnetosphere, Journal of Geophysical Research: Space Physics, 123(7), 5528-5537, doi:10.1029/2018JA025637.

Zimbardo, G., E. Amato, A. Bovet, F. Effenberger, A. Fasoli, H. Fichtner, I. Furno, K. Gustafson, P. Ricci, S. Perri, and et al. (2015), Superdiffusive transport in laboratory and astrophysical plasmas, Journal of Plasma Physics, 81(6), 495810,601, doi: $10.1017 /$ S0022377815001117. 

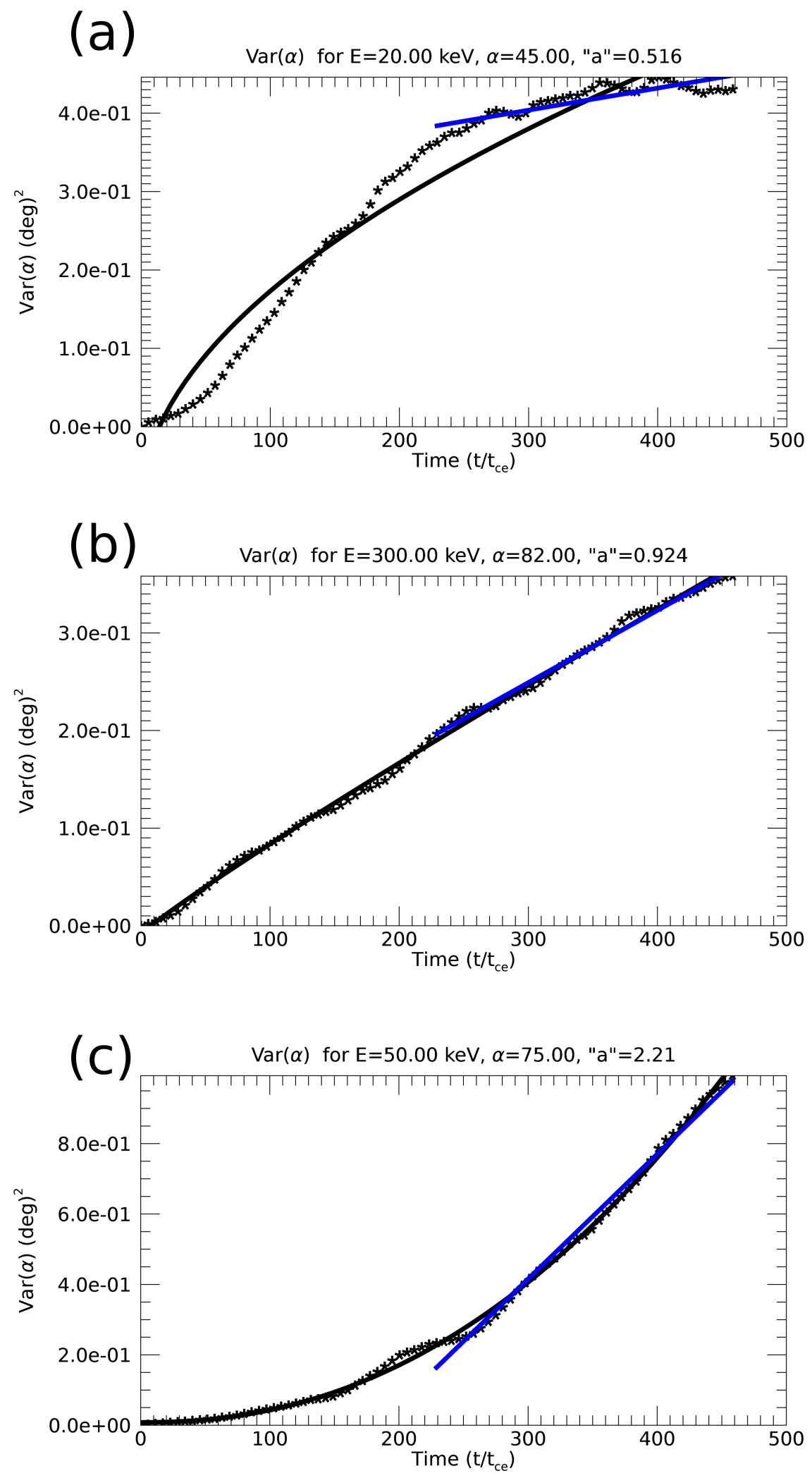

Figure 7. $\operatorname{var}\left(\alpha_{l}\right)$ for three bins roughly centred on: (a): $(E, \alpha)=\left(20 \mathrm{keV}, 45^{\circ}\right),(\mathrm{b}):(E, \alpha)=\left(300 \mathrm{keV}, 82^{\circ}\right)$, and (c): $\left(50 \mathrm{keV}, 75^{\circ}\right)$ respectively. Asterisks mark the particle data, and solid black lines mark curve fits. The solid blue line is a linear fit over $T / 2<\tilde{t}<T$. 


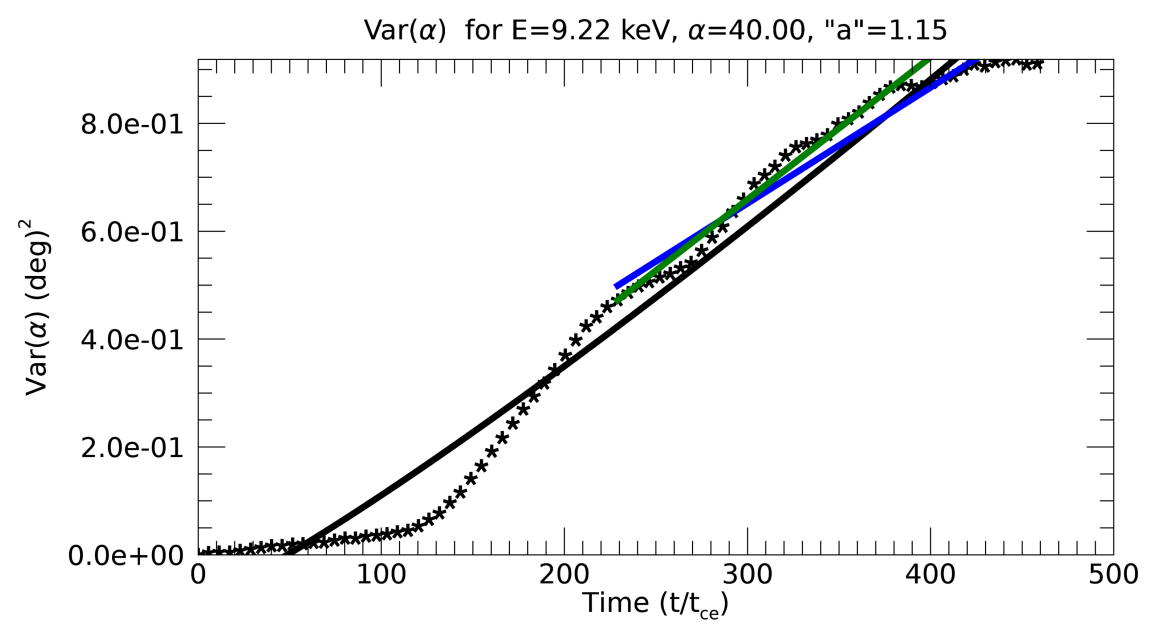

Figure 8. $\operatorname{var}\left(\alpha_{l}\right)$ for a bin roughly centred on $(E, \alpha)=\left(9.2 \mathrm{keV}, 40^{\circ}\right)$. Asterisks mark the particle data, solid black lines is a curve fits, and the solid blue line is a linear fit over $T / 2<\tilde{t}<T$. The solid green line shows evolution of $\operatorname{var}(\alpha)$ consistent with the PADIE diffusion coefficient, $D_{\alpha \alpha}$, over $T / 2<\tilde{t}<T$. 


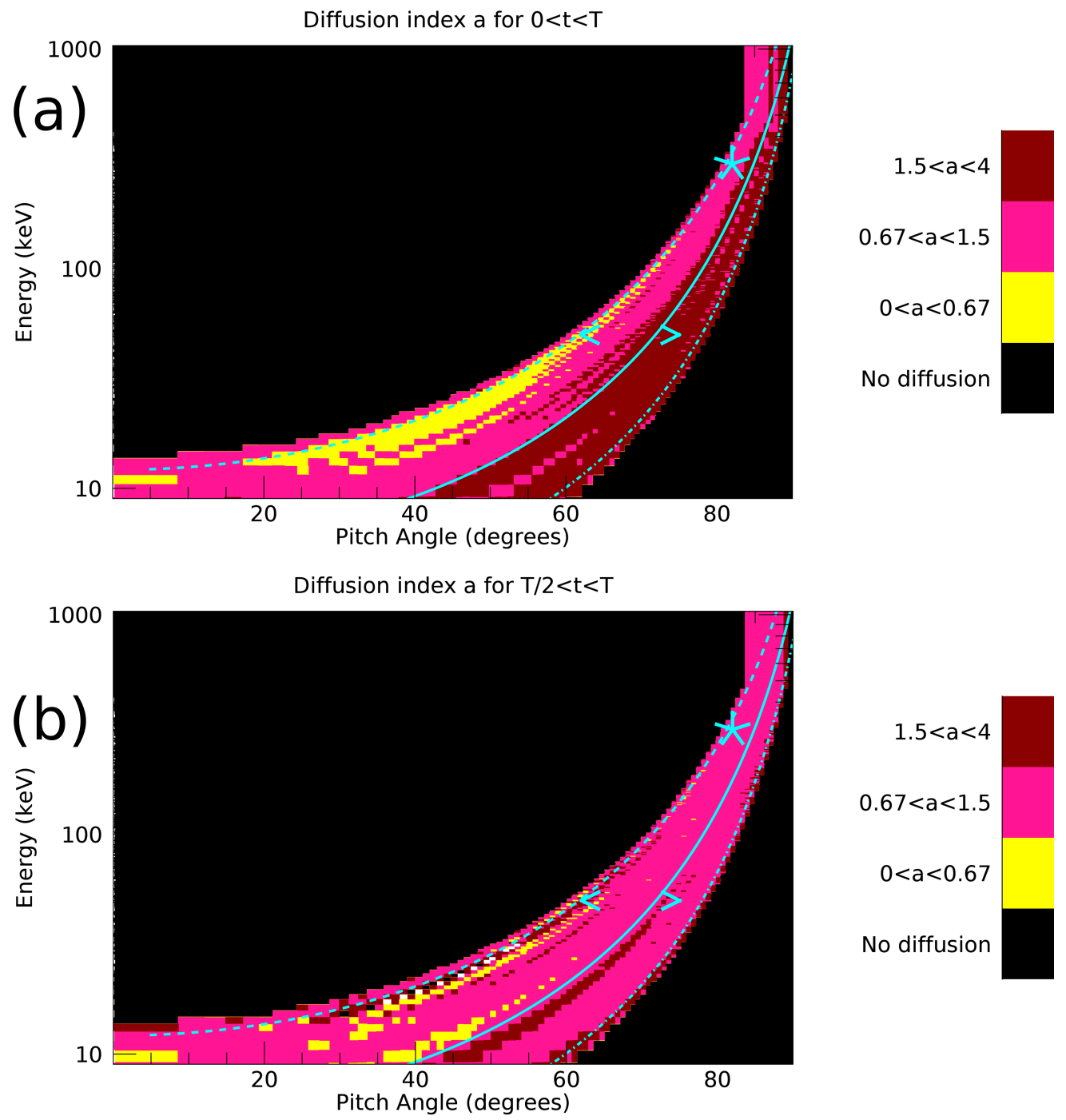

Figure 9. The diffusion index, $a$, for each $(E, \alpha)$ bin in the experiment. Plot(a) shows this index over the entire time of wave-particle interaction $(0<\tilde{t}<T)$, whereas plot (b) shows the index for the second half of the interaction only $(T / 2<\tilde{t}<T)$. Black indicates 'no diffusion'. Yellow indicates 'sub-diffusion' $(0<a<0.67)$, pink indicates normal diffusion $(0.67<a<1.5)$, and dark red regions indicate superdiffusion $(a>1.5)$. The over-plotted cyan curves mark the values of energy and pitch angle that are (according to equations (4) and (5)) in " $n=-1$ resonance" with waves of frequency $f_{l c}$ ('dash'), $f_{u c}$ ('dash-dot'), and $0.5\left(f_{l c}+f_{u c}\right)$ ('solid'). The bins represented in figure 7(a)-(c) are marked by cyan symbols: "<", " " and ">". 
Figure 1. 
$\longrightarrow \mathrm{F}(\mathrm{t})=$ Energy $($ Total, $\mathrm{t})-\operatorname{Energy}\left(\mathrm{B}_{0}\right)-\int_{\tau=0}^{\tau=\mathrm{t}} \int_{\mathrm{V}} \nabla \cdot \mathrm{S}(\mathrm{x}, \tau) \mathrm{dVd} \tau$ $F(t)=\operatorname{Energy}\left(e^{-}, t\right)$

- - - - $\mathrm{F}(\mathrm{t})=\operatorname{Energy}(\delta \mathrm{E} \delta \mathrm{B}, \mathrm{t})$

$\int_{\tau=0}^{\tau=\mathrm{t}} \int_{\mathrm{V}} \nabla \cdot \mathrm{S}(\mathrm{X}, \tau) \mathrm{dVd} \tau$

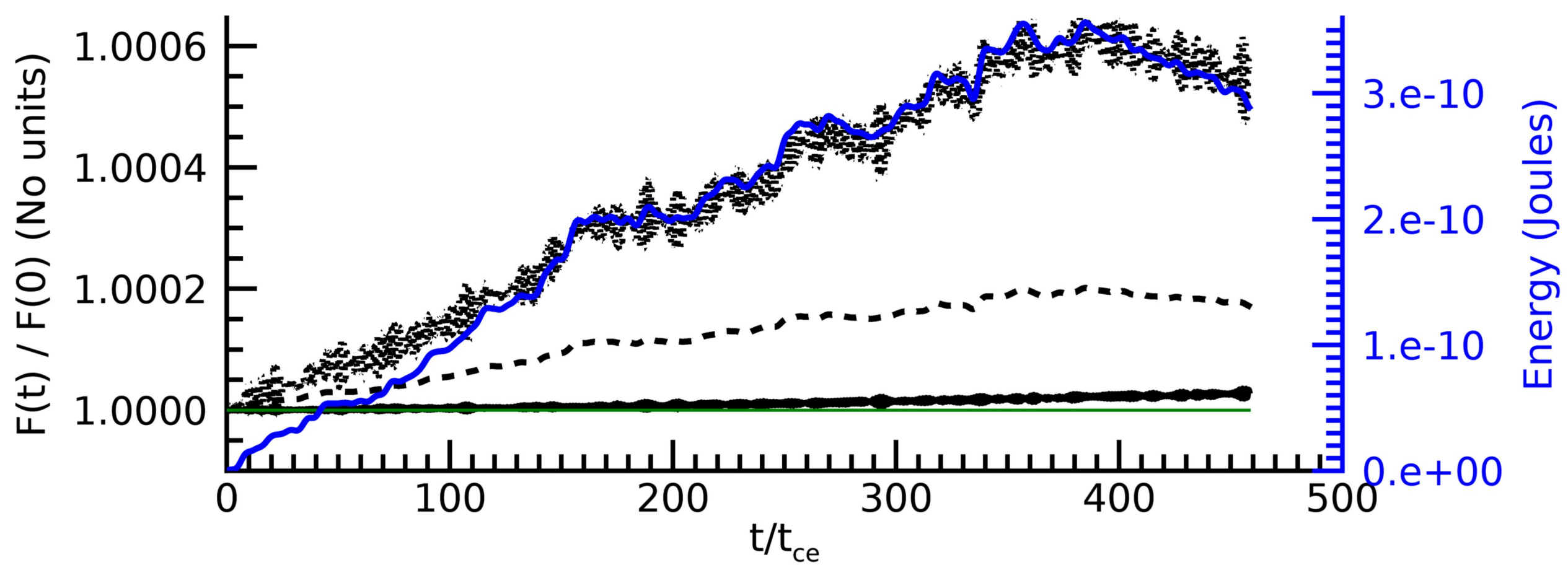


Figure 2. 


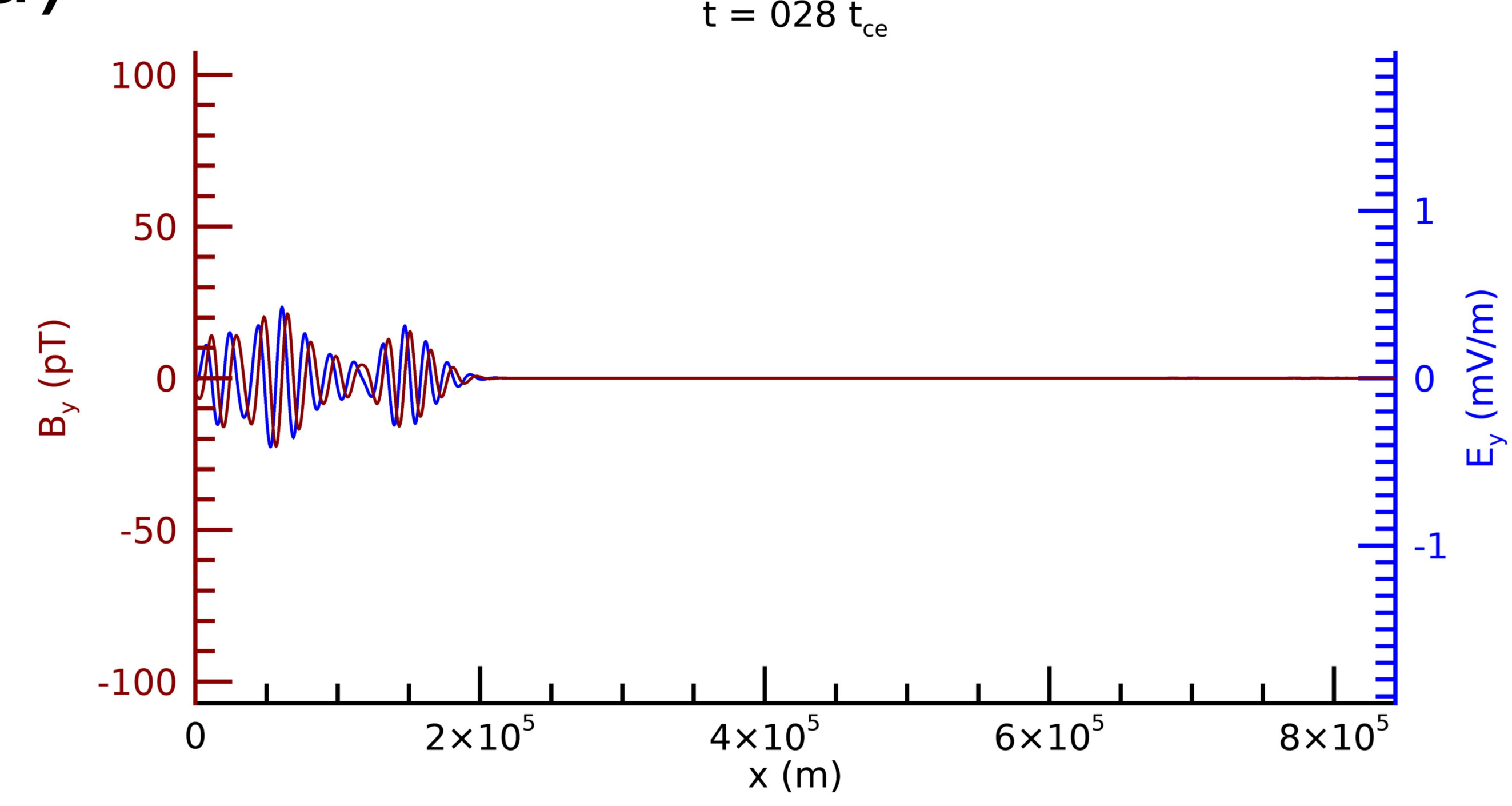

(c)

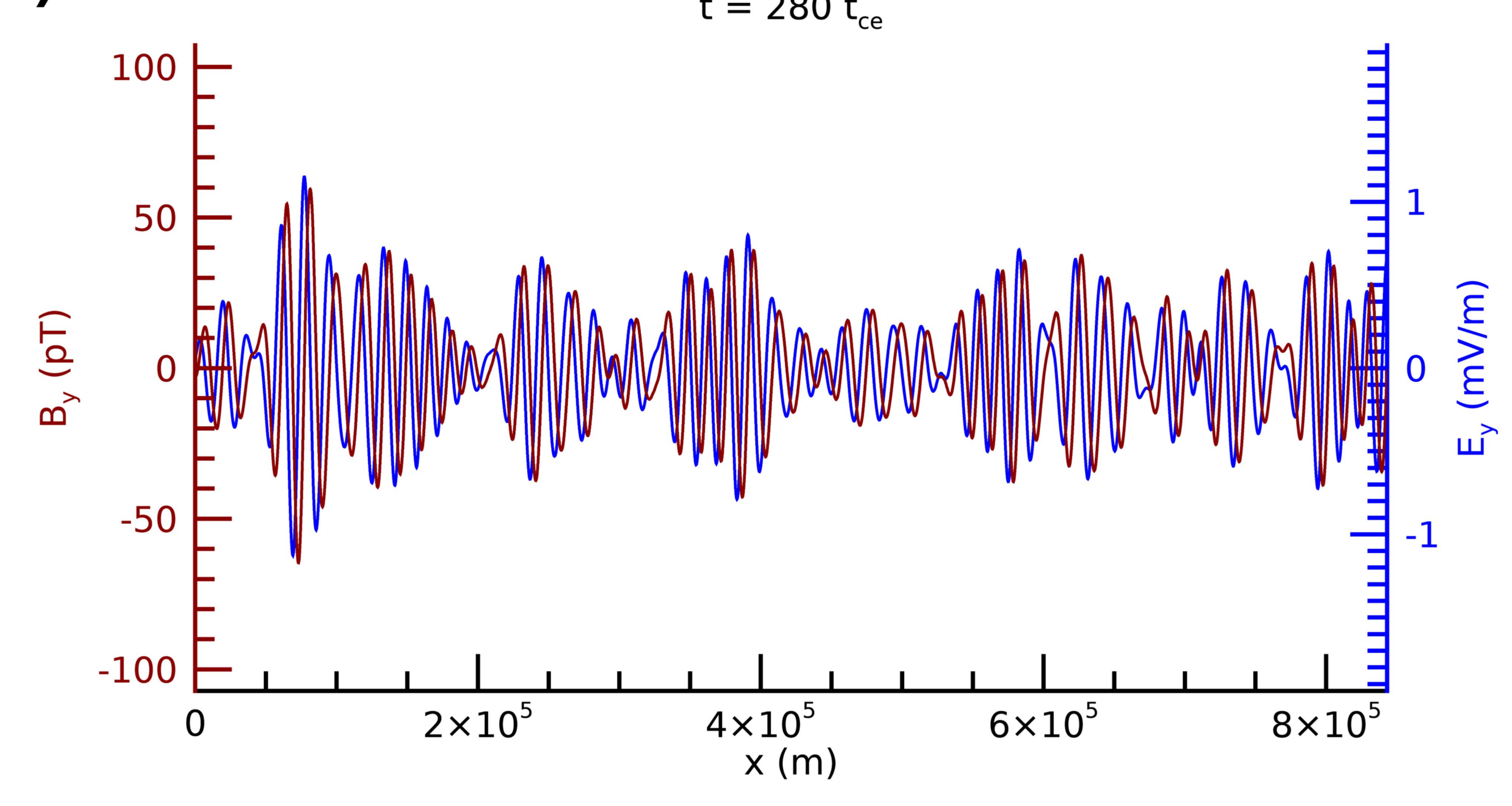

a)

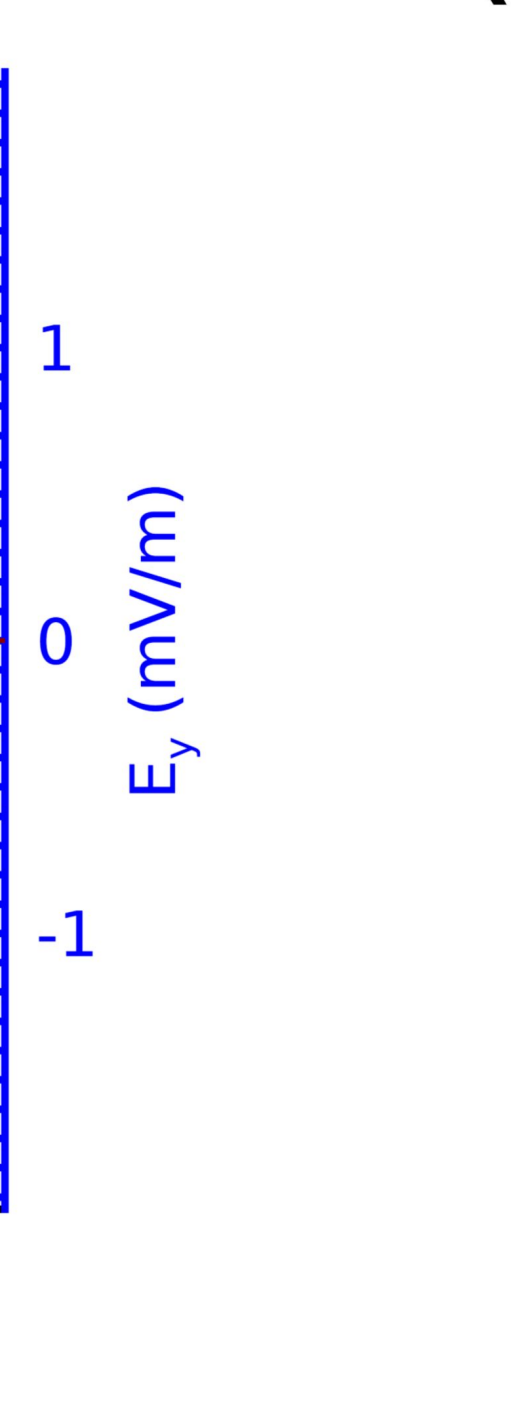

.

(d)

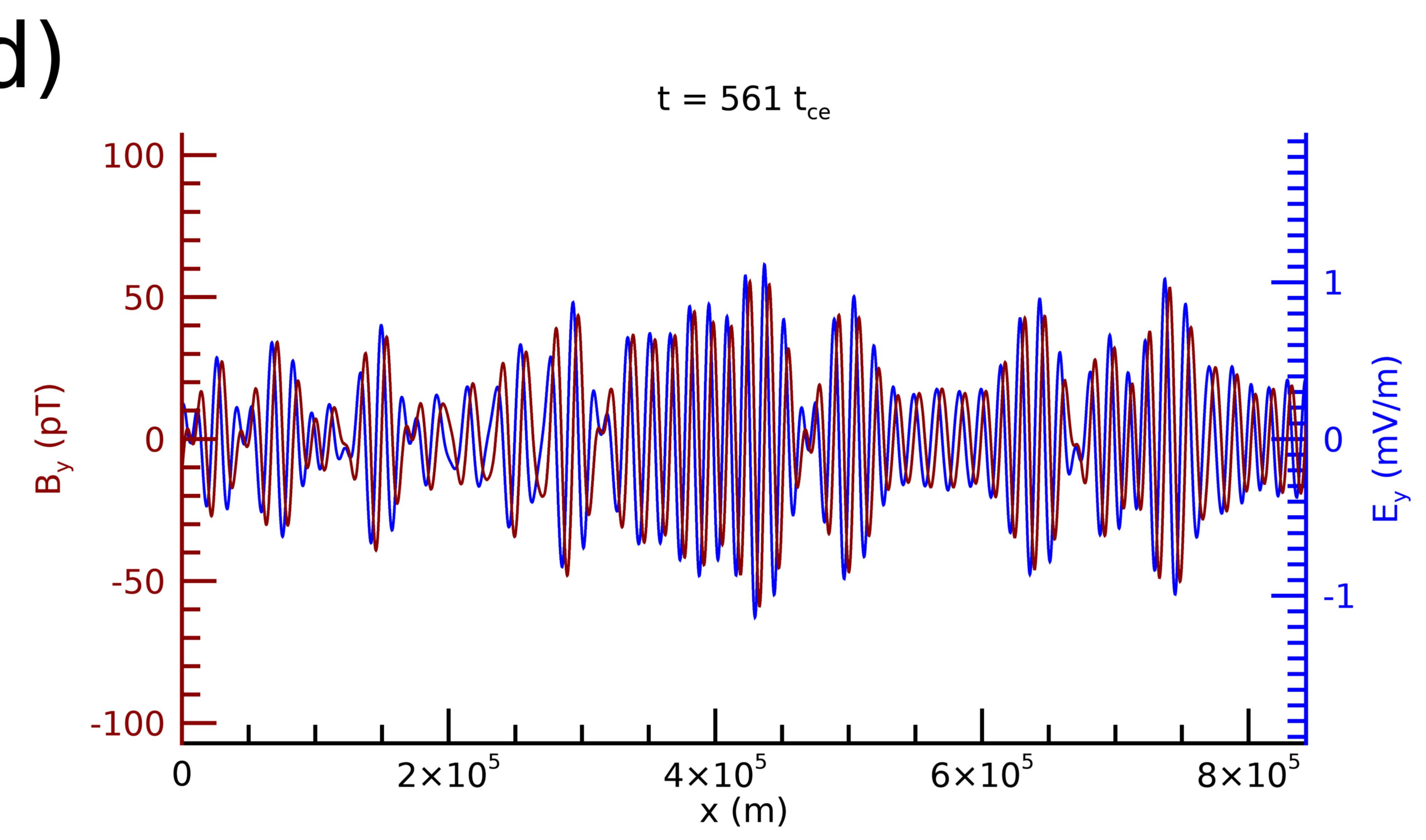

(d)

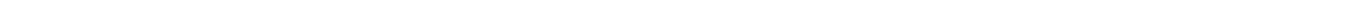

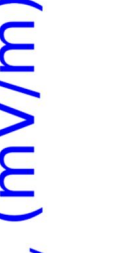

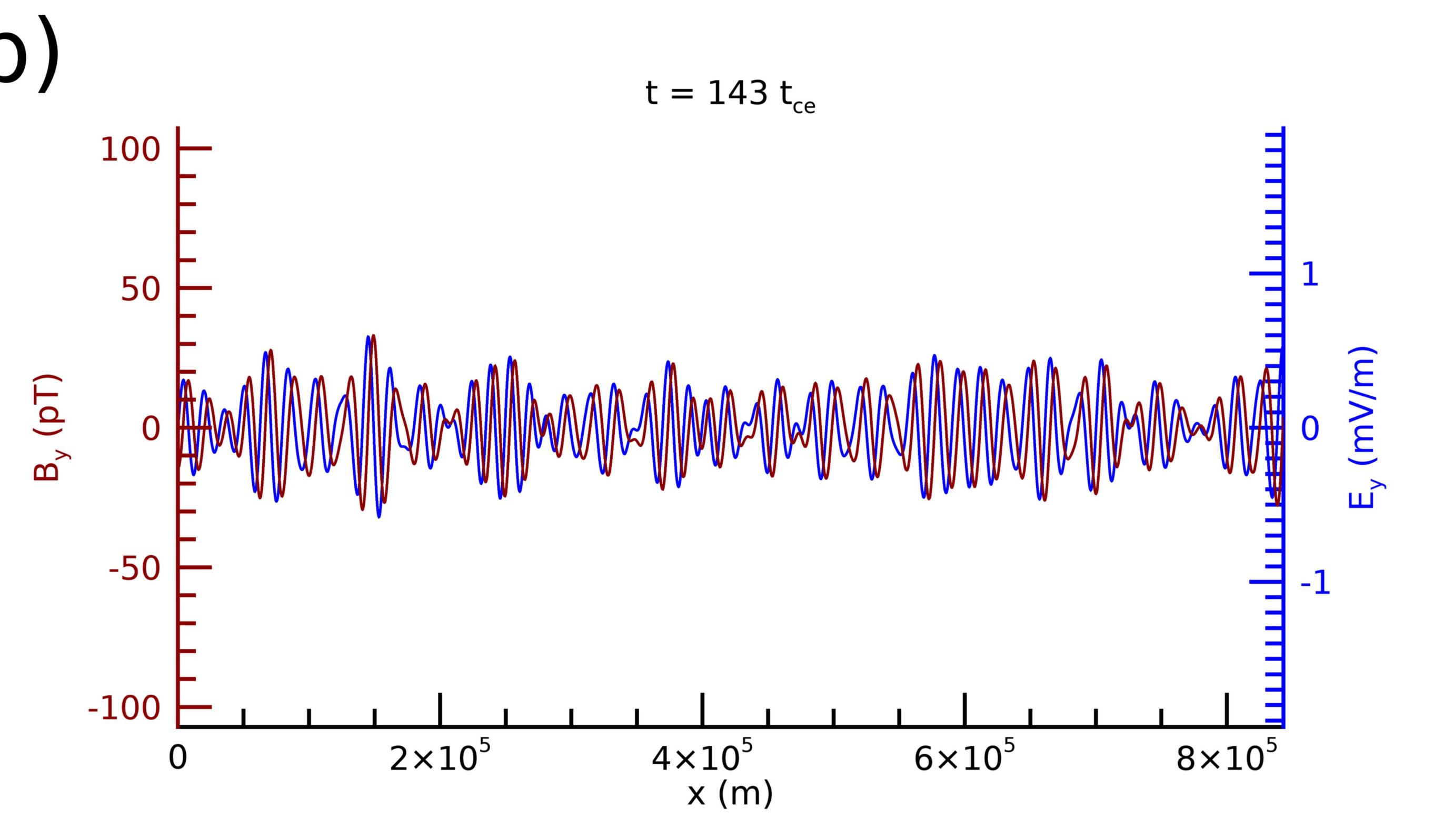

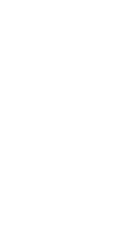


Figure 3. 
Fourier Amplitude of $\mathrm{E}_{\mathrm{y}}$ and $\mathrm{B}_{\mathrm{y}}$ components

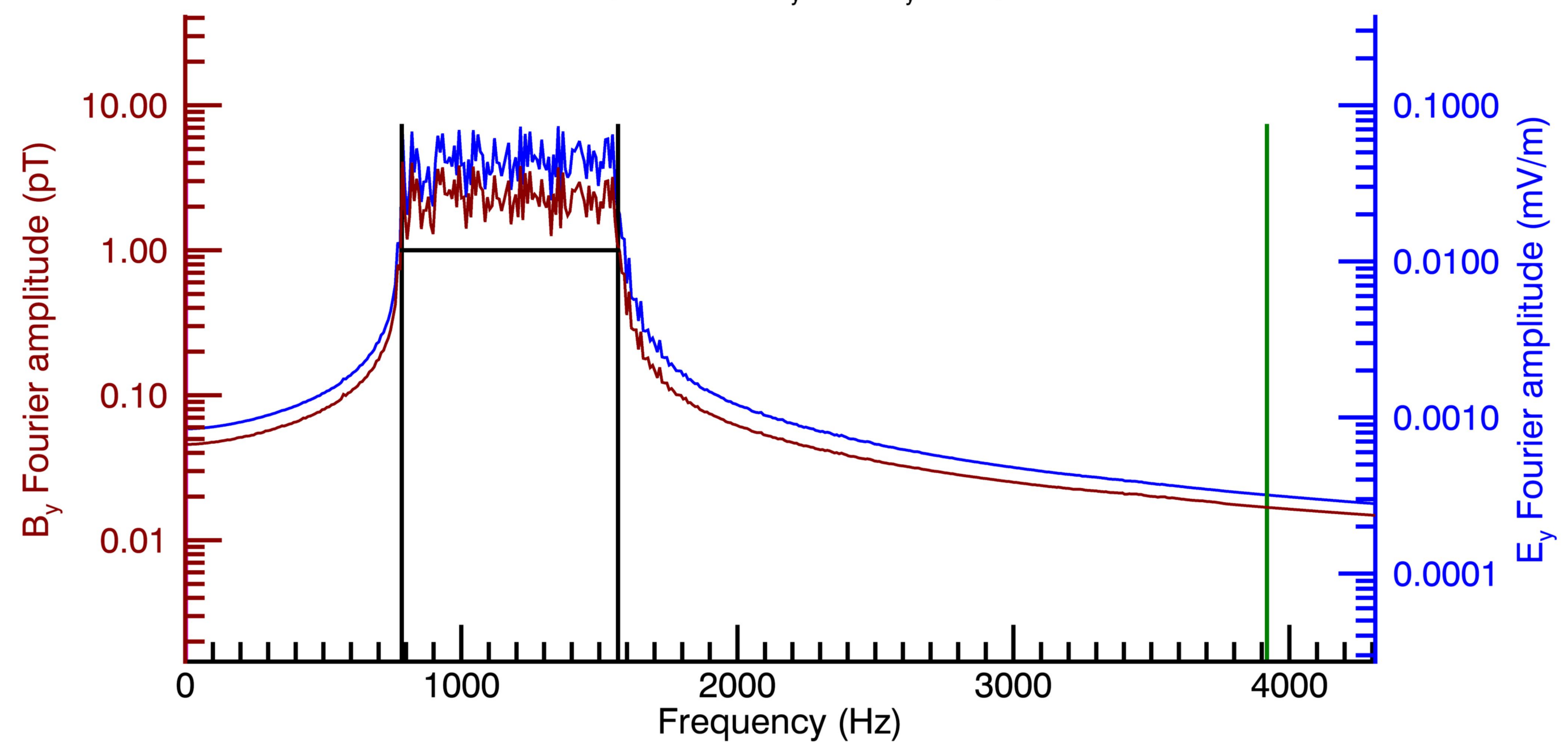


Figure 4. 
Dispersion relation for $B_{y}$

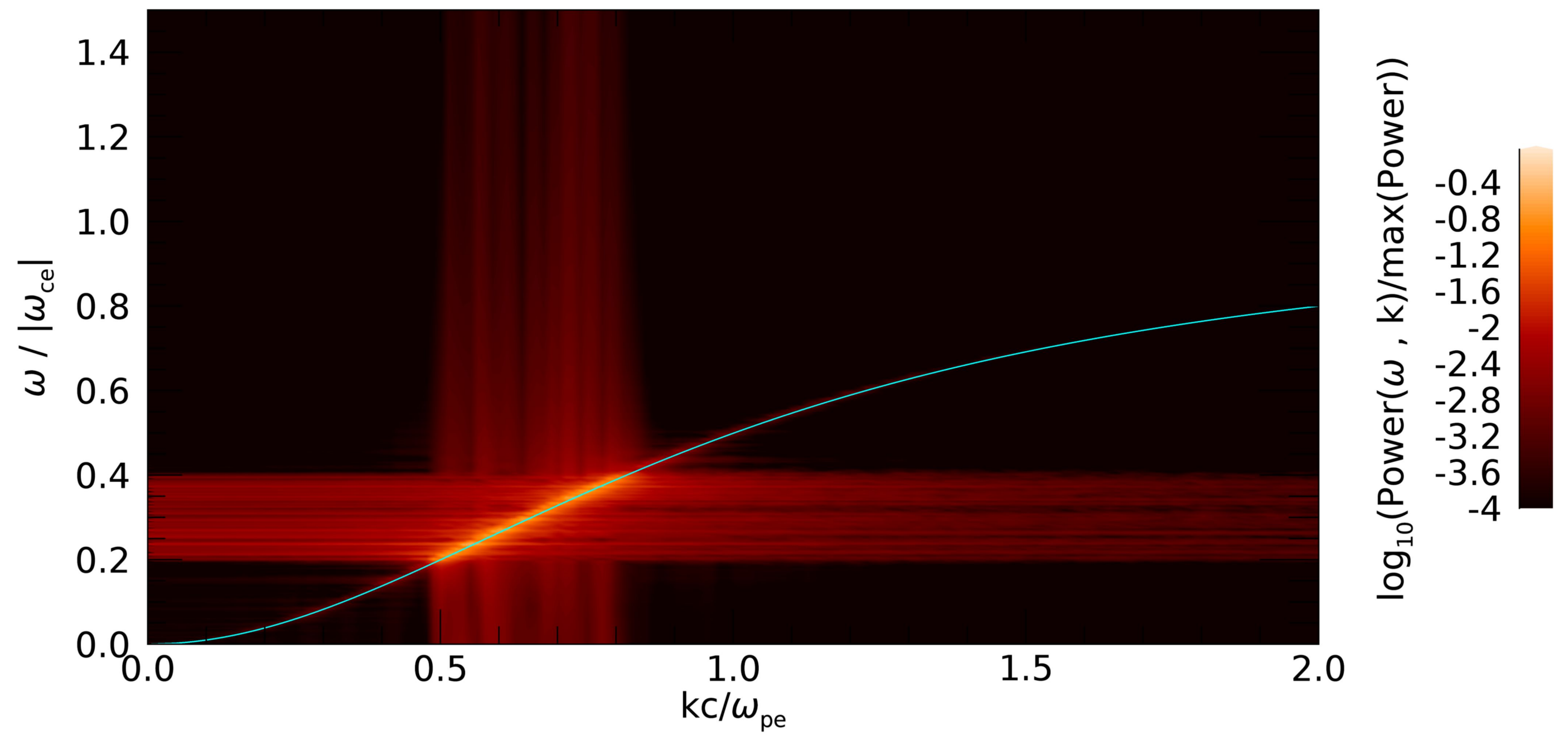


Figure 5. 
Figure 6. 


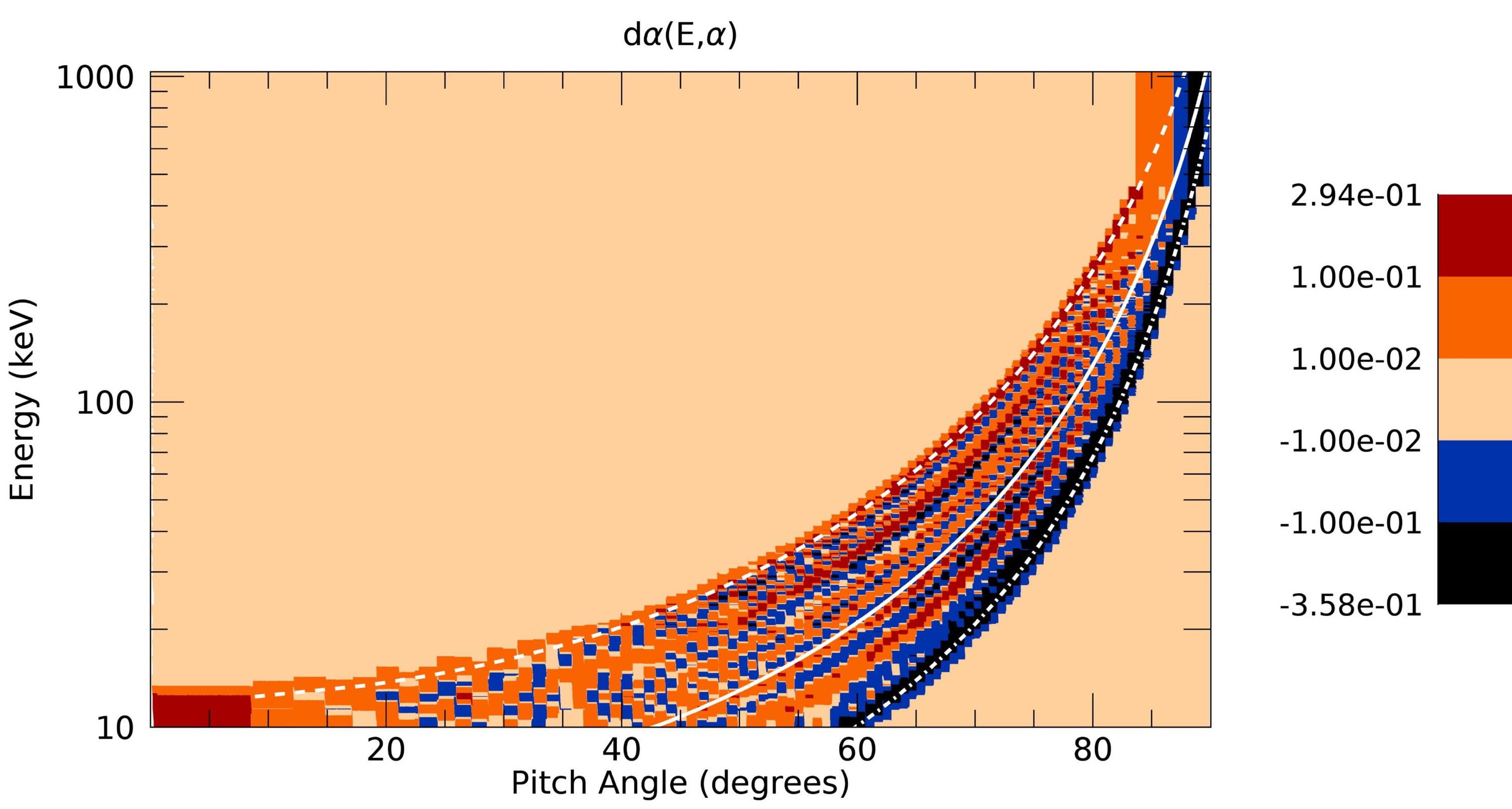


Figure 7. 
Figure 8. 


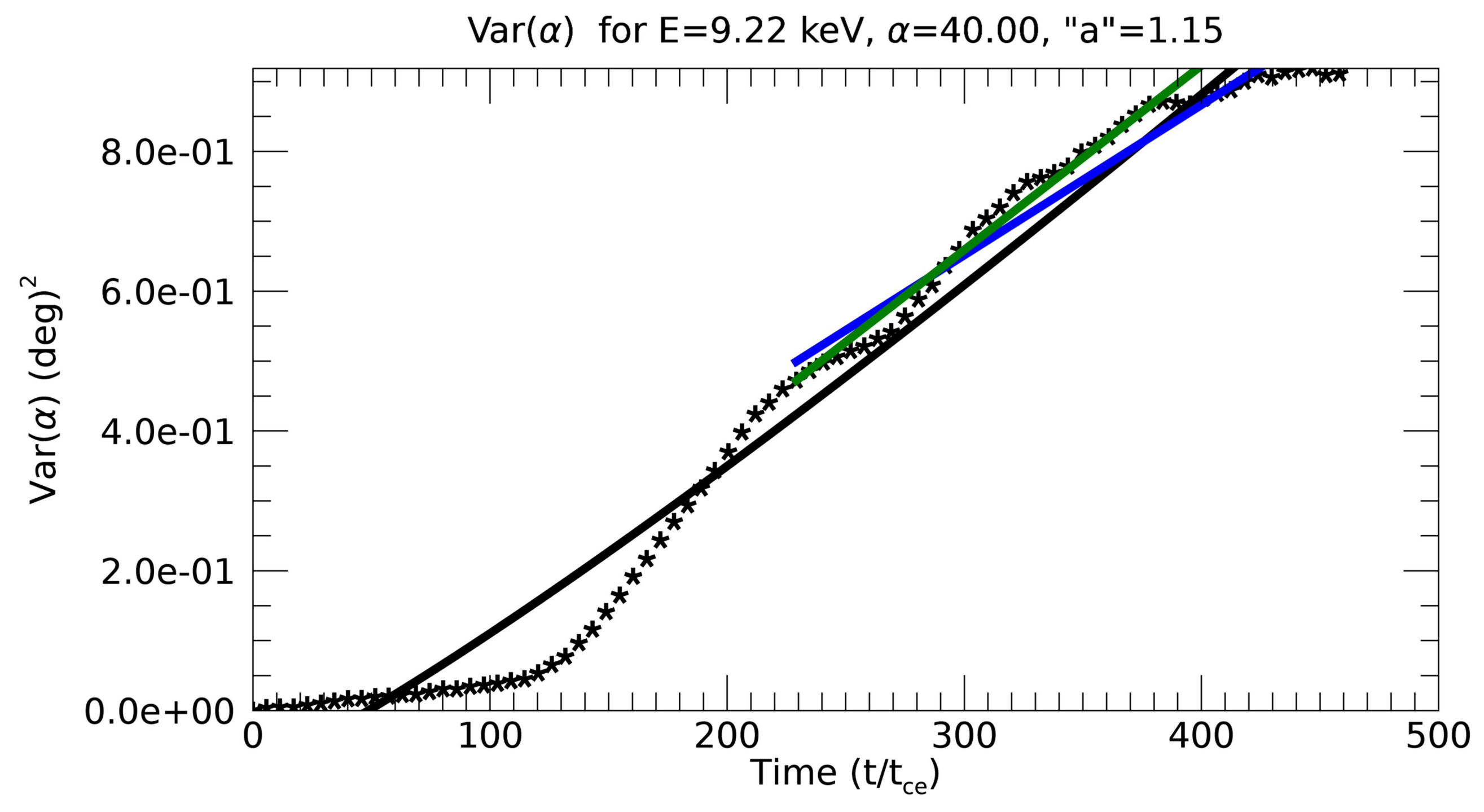


Figure 9. 
Diffusion index a for $0<t<T$

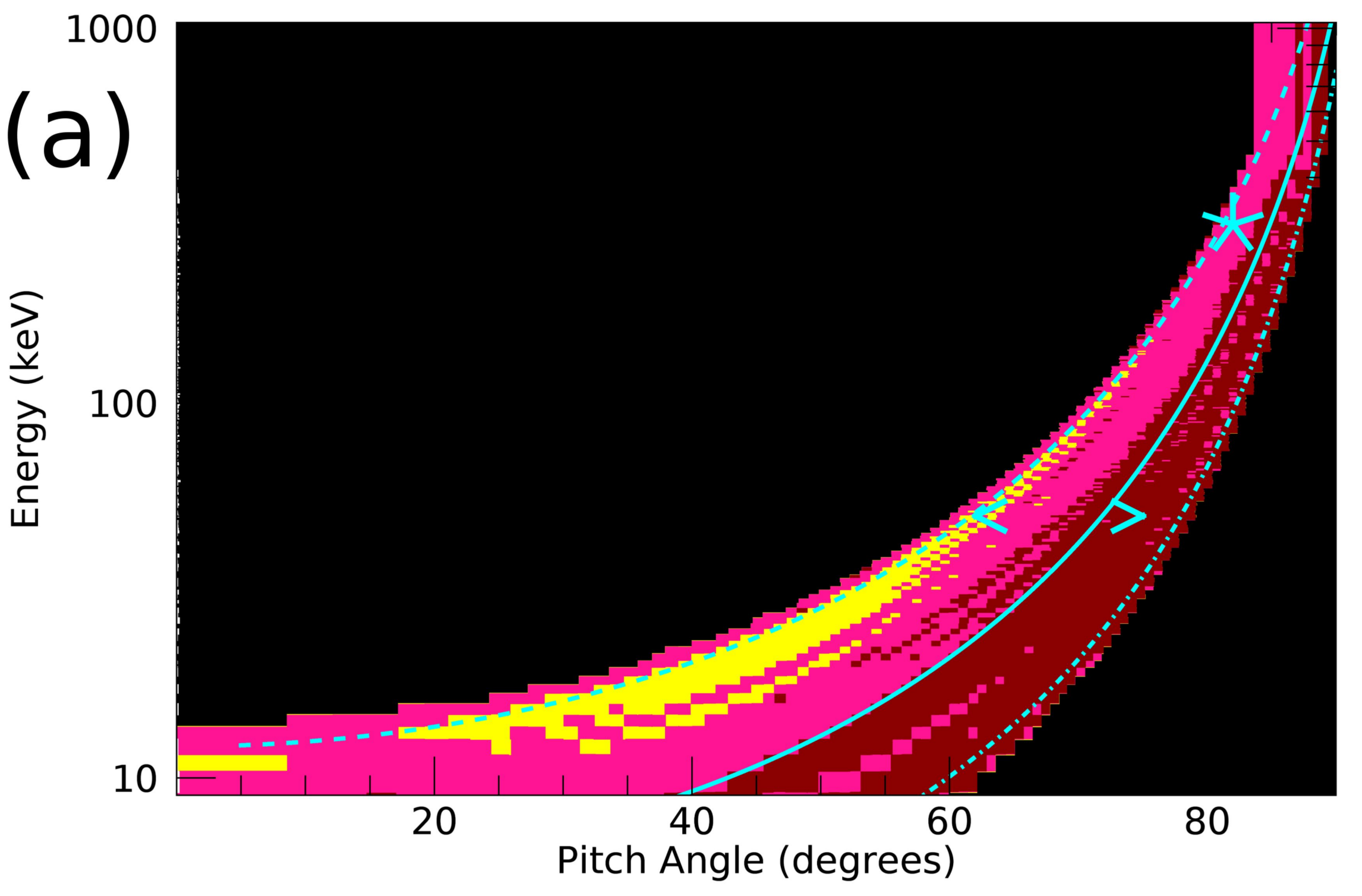

No diffusion

Diffusion index a for $\mathrm{T} / 2<\mathrm{t}<\mathrm{T}$

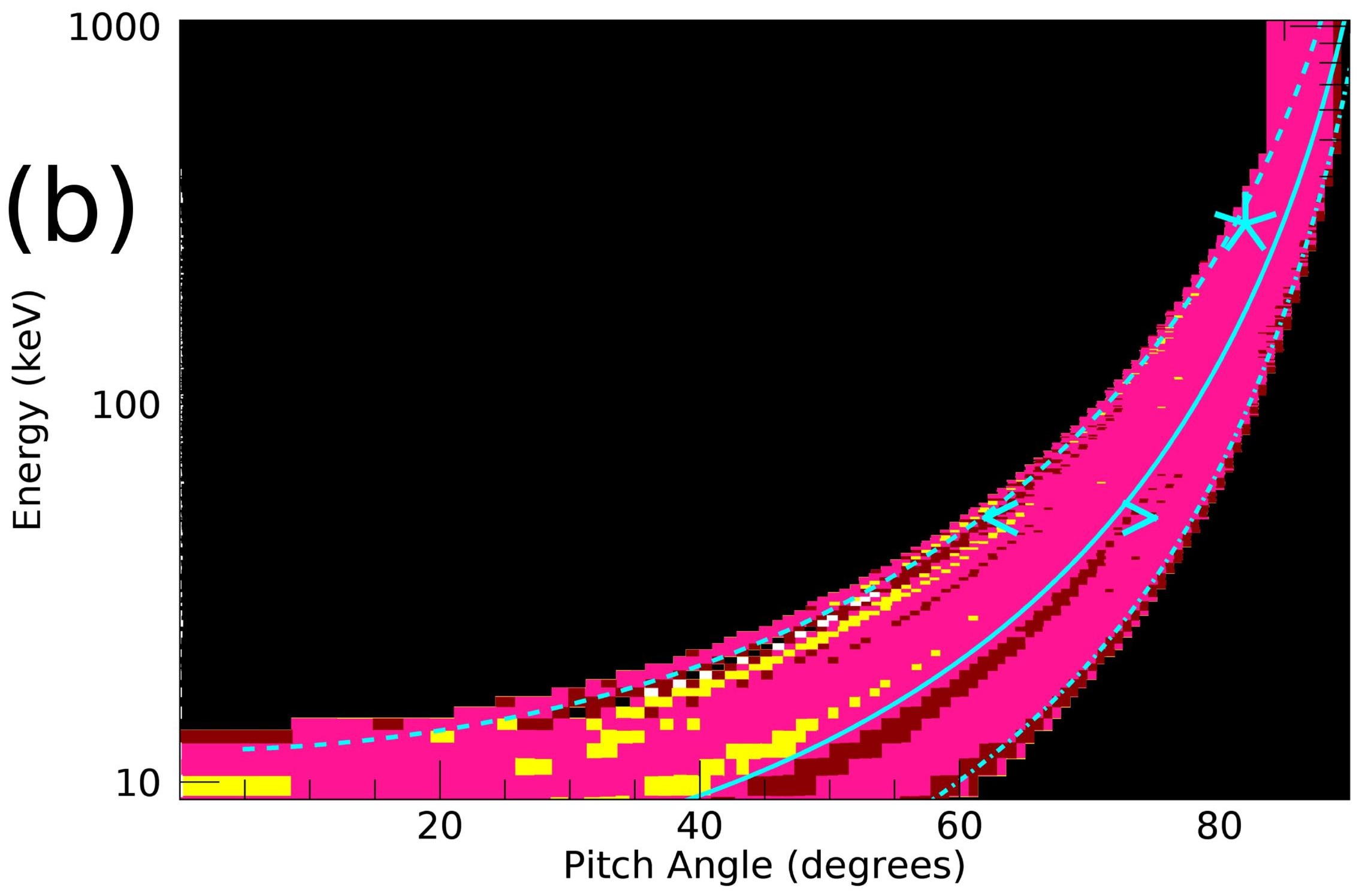

